\title{
Theoretical mass, liquid, and polymer sensitivity of acoustic wave sensors with viscoelastic guiding layers
}

\author{
Glen McHale, ${ }^{\text {a) }}$ Michael lan Newton, and Fabrice Martin \\ Department of Chemistry and Physics, The Nottingham Trent University, Clifton Lane, \\ Nottingham NG11 8NS, United Kingdom
}

(Received 12 July 2002; accepted 4 October 2002)

\begin{abstract}
The theoretical sensitivity of Love wave and layer-guided shear horizontal acoustic plate mode (SH-APM) sensors for viscoelastic guiding layers and general loading by viscoelastic materials is developed. A dispersion equation previously derived for a system of three rigidly coupled elastic mass layers is modified so that the second and third layers can be viscoelastic. The inclusion of viscoelasticity into the second, wave guiding layer, introduces a damping term, in addition to a phase velocity shift, into the response of the acoustic wave system. Both the waveguiding layer and the third, perturbing layer, are modeled using a Maxwell model of viscoelasticity. The model therefore includes the limits of loading of both nonguided shear horizontal surface acoustic wave and acoustic plate mode (APM) sensors, in addition to Love wave and layer-guided SH-APM sensors, by rigidly coupled elastic mass and by Newtonian liquids. The three-layer model is extended to include a viscoelastic fourth layer of arbitrary thickness and so enable mass deposition onto an immersed Love wave or layer-guided SH-APM sensor to be described. A relationship between the change in the complex velocity and the slope of the complex dispersion curve is derived and the similarity to the mass and liquid sensor response of quartz crystal microbalances is discussed. Numerical calculations are presented for the case of a Love wave device in vacuum with a viscoelastic waveguiding layer. It is shown that, while a particular polymer relaxation time may be chosen such that the effect of viscoelasticity on the real part of the phase speed is relatively small, it may nonetheless induce a large insertion loss. The potential or the use of insertion loss as a sensor parameter is discussed. (C) 2003 American Institute of Physics. [DOI: 10.1063/1.1524309]
\end{abstract}

\section{INTRODUCTION}

A wide range of acoustic wave sensors have been reported in the literature for use as mass sensors. ${ }^{1-3}$ When the mass being sensed is deposited from the liquid phase or the focus of the application is to sense the properties of a liquid phase, the most obvious choice of acoustic wave mode is one with a shear horizontal polarization to the displacement. This is because, for most acoustic wave devices, an out-of-plane motion would induce a compressional (sound) wave in the liquid and so cause high damping. ${ }^{4,5}$ The exception to this occurs for flexural plate wave devices where the wave speed is less than the speed of sound in the liquid so that compressional wave generation does not occur even though an outof-plane displacement exists. One of the most common shear mode type of sensors is the quartz crystal microbalance (QCM) and this has been extensively used for chemical and biochemical studies. The QCM has the advantage of simplicity because it is a simple thickness shear mode oscillation where the crystal thickness determines the resonant frequency and the transducers are simple metallizations of the upper and lower crystal faces. Deposition of a thin mass layer onto a QCM operated in its fundamental mode causes a frequency shift proportional to the mass per unit area and the

\footnotetext{
a) Author to whom correspondence should be addressed: electronic mail: glen.mchale@ntu.ac.uk
}

square of the operating frequency, but does not cause any attenuation of the oscillation. This result, summarized by the Sauerbrey equation, ${ }^{6}$ can be shown to be valid, at least approximately, even when the mass is deposited from the liquid phase. ${ }^{7}$ Introducing a QCM from vacuum into a Newtonian liquid results in both a frequency shift and an attenuation of the resonance. The effect of the shear mode oscillation is to entrain fluid within a penetration depth $\delta=\left(2 \eta_{f} / \omega \rho_{f}\right)^{1 / 2}$ of the surface, where $\eta_{f}$ is the fluid's viscosity, $\rho_{f}$ is the fluid's density, and $\omega$ is the angular frequency. The oscillation in the liquid decays within a penetration and so the QCM can be viewed as sensing the interfacial mass defined by the penetration depth. Since the penetration depth depends on the inverse of the square root of frequency, the frequency shift on immersion in a Newtonian liquid becomes proportional to the frequency to the power of $3 / 2$ rather than squared. In addition, the frequency shift is proportional to the square root of the viscosity-density product. These conclusions for liquid phase sensing were described by Kanazawa and Gordon. ${ }^{8}$ The difficulty with the QCM as a sensor for biological applications is that high sensitivity is needed and this requires higher fundamental frequency and consequently thinner and more fragile crystals.

An alternative to the QCM is to use a shear mode surface acoustic wave (SAW) type sensor. In such sensors, the operating frequency is determined by the spacing between the fingers in a surface fabricated interdigital transducer to- 
gether with the mode speed determined by the substrate type and propagation axis. ${ }^{9-11}$ Shear horizontal (SH) SAWs and acoustic plate mode (APMs) have been considered by some workers, but it has been claimed that higher mass sensitivity can be obtained by using a waveguiding layer on the surface of a SH-SAW to create a Love wave device. ${ }^{12,13}$ Experimentally, Love wave devices have been created using substrates supporting surface skimming bulk waves (SSBWs) or SHSAWs and the waveguiding layer have been materials such as silicon dioxide $\left(\mathrm{SiO}_{2}\right)$ or poly(methylmethacrylate). ${ }^{14,15}$ In our previous work we have used both types of substrate and a range of polymer photoresists. ${ }^{16}$ While much experimental work has been reported by both ourselves and others using these types of systems, most theoretical considerations of Love waves assume a rigidly coupled elastic mass guiding layer and an infinitely thick substrate. It is evident from the insertion loss that occurs in experiments, but which is not predicted by Love wave theory based on a rigidly coupled elastic mass guiding layer, that theoretical work on the effects of viscoelasticity of waveguiding layers is needed. It is also highly relevant to note that outside of the biosensing field, the use of molecularly imprinted polymers (MIPs) as both waveguide and analyte selective layers in Love wave devices for vapor phase sensing has been reported. In our own work we have used MIPs as coatings on QCMs to detect a range of analytes, including steroids in the liquid phase. ${ }^{17,18}$ Thus, the experimental motivation to develop models for the effect of viscoelasticity on acoustic wave sensors is urgent.

In our previous theoretical work we have shown that SH-APMs can be viewed within the same theoretical framework as Love waves. ${ }^{19-21}$ This involved extending the theoretical treatment of both Love wave sensors with guiding layers composed of elastic mass to Love waves on finite thickness substrates and of SH-APMs to SH-APMs coated by waveguiding layers. In this previous treatment, higher order Love wave modes were shown to be continuations of the layer-guided SH-APMs and it was shown that significantly enhanced mass sensitivity could be obtained for SHAPMs by the use of a waveguiding layer. In addition, a rigorous relationship was derived relating the slope of the dispersion curve to the mass sensitivity of a Love wave or layer guided SH-APM mode. This relationship between the slope of the dispersion curve and the mass sensitivity is of importance because it allows an experimentally determined dispersion curve to be used to predict the mass sensitivity of a sensor and to predict the change in sensitivity with frequency. In the present article, we significantly extend our previous formalism to include the effects of viscoelasticity. In order to provide as wide a range of applicability as possible we consider the effect of viscoelasticity of both the waveguiding layer and the material being sensed. This treatment therefore describes the following situations:

(1) SH-SAW and SH-APM perturbation responses to viscoelastic layers of finite or infinite thickness;

(2) dispersion curves for Love wave and layer-guided SHAPMs when the guiding layer is viscoelastic;
(3) response of Love wave and layer-guided SH-APMs to perturbing viscoelastic layers of finite or infinite thickness; and

(4) response of Love wave and layer-guided SH-APMs to mass deposition from the liquid phase.

In each of the above cases of viscoelasticity, we also give results for the limits of thin elastic mass layers and for infinite thickness of Newtonian liquids. In addition, the relationship between the slope of the dispersion curve and the response of a Love wave or layer-guided sensor is generalized to include both viscoelastic waveguide layers and viscoelastic perturbations. Thus, the present article is sufficiently detailed mathematically to provide comprehensive analytical results for the velocity shift and attenuation response of SHSAW, SH-APM, Love wave, and layer-guided SH-APM devices as sensors in the gas and liquid phases.

The organization of the article begins with a brief review of the three-layer model describing a substrate, guiding layer, and perturbing layer, all composed of rigidly coupled elastic mass. Then, the idea of viscoelasticity for a layer is introduced and a complex shear modulus defined. It is shown how the Navier-Stokes equations describing a viscoelastic layer can be transformed into a wave equation and how the previous results for the three-layer model can be extended. In addition, the equations describing the viscoelasticity using a relaxation time and the Maxwell model are introduced and the relationship to the penetration depth and mode speed in the three-layer model is defined. Subsequently, the analytical treatment of a viscoelastic guiding layer is developed with the important limiting cases of SH-SAW and SH-APM sensor response to mass, Newtonian liquid, and viscoelastic liquid loading detailed. For the general viscoelastic guiding layer a relationship between the complex velocity (i.e., velocity shift and insertion loss) and the slope of the dispersion curve is developed. For completeness, a four-layer model is also developed so that the response of an immersed device to mass deposition can be considered. The relationship of our Love wave and layer-guided SH-APM results to the Sauerbrey and Kanazawa results are detailed. Finally, we conclude by focusing on numerical calculations for the effects of viscoelastic guiding layers on Love wave devices and, in particular, on the consequences for sensors of the insertion loss.

\section{BASIC THEORETICAL FORMULATION}

\section{A. Three layers of rigidly coupled elastic mass}

In a previous article we considered the propagation of shear horizontally polarized acoustic waves in a system composed of a substrate of thickness $w$ overlayed by a waveguiding layer of thickness $d$ and an additional mass layer of thickness $h$ (Fig. 1). In this section, the theoretical development is briefly reviewed so that modifications due to viscoelasticity can be clearly identified. The substrate and layers were considered to be rigidly coupled elastic mass layers and the equation of motion in each material reduced to

$$
\rho \frac{\partial^{2} u_{j}}{\partial t^{2}}=\mu \nabla^{2} u_{j}
$$




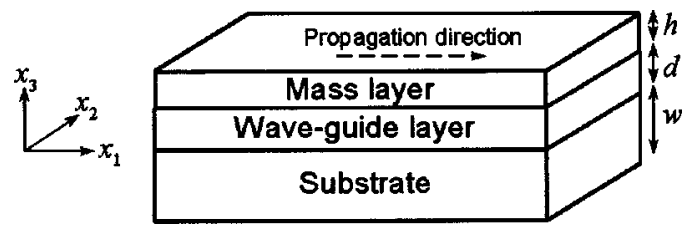

FIG. 1. Definition of axes, symbols, and layer parameters for propagation of shear horizontally polarized acoustic waves in a three-layer system. For sensor applications the first layer is the substrate, the second layer is the waveguiding layer, and the third layer is the perturbing layer.

where $\rho$ is the density of the material and $\mu$ is the shear modulus. The equation of motion Eq. (1) was then solved in each material using trial solutions of the form

$$
\begin{aligned}
& u_{s}=(0,1,0)\left\lfloor C_{s} e^{-T_{s} x_{3}}+D_{s} e^{T_{s} x_{3}}\right\rfloor e^{j\left(\omega t-k_{1} x_{1}\right)}, \\
& u_{l}=(0,1,0)\left\lfloor A_{l} e^{-j T_{l} x_{3}}+B_{l} e^{j T_{l} x_{3}}\right\rfloor e^{j\left(\omega t-k_{1} x_{1}\right)}, \\
& u_{p}=(0,1,0)\left[E_{p} e^{-j T_{p} x_{3}}+F_{p} e^{j T_{p} x_{3}}\right] e^{j\left(\omega t-k_{1} x_{1}\right)},
\end{aligned}
$$

where the subscripts $s, l$ and $p$ indicate substrate, guiding layer, and perturbing layer and $k_{1}=(\omega / \nu)$ gives the phase speed $\nu$ of the solution. The forms of Eqs. (2)-(4) were chosen for their similarity to the displacements of a Love wave solution, but the wave vector $T_{s}$ can become imaginary and can therefore also represent a shear horizontal acoustic plate mode with a guiding and/or a mass layer. Substituting the trial solutions into the equations of motion for the materials gives the following conditions on the wave vectors:

$$
\begin{aligned}
& T_{s}^{2}=\omega^{2}\left(\frac{1}{\nu^{2}}-\frac{1}{\nu_{s}^{2}}\right), \\
& T_{l}^{2}=\omega^{2}\left(\frac{1}{\nu_{l}^{2}}-\frac{1}{\nu^{2}}\right), \\
& T_{p}^{2}=\omega^{2}\left(\frac{1}{\nu_{p}^{2}}-\frac{1}{\nu^{2}}\right) .
\end{aligned}
$$

For elastic mass the density and shear modulus define the shear speed of the layers by $\nu_{s}=\left(\mu_{s} / \rho_{s}\right)^{1 / 2}, \quad \nu_{l}$ $=\left(\mu_{l} / \rho_{l}\right)^{1 / 2}$, and $\nu_{p}=\left(\mu_{p} / \rho_{p}\right)^{1 / 2}$. The final requirement was that the solutions in each material should satisfy boundary conditions of continuity of displacement and stress at the boundaries; the stress is given by

$$
T_{i 3}=\delta_{i 2} \mu\left(\frac{\partial u_{2}}{\partial x_{3}}\right) .
$$

Applying the boundary conditions gave a dispersion equation for a three-layer system

$$
\begin{aligned}
\tan \left(T_{l} d\right)= & \xi \tanh \left(T_{s} w\right)-\xi_{p} \tan \left(T_{p} h\right) \\
& \times\left[1+\xi \tan \left(T_{l} d\right) \tanh \left(T_{s} w\right)\right]
\end{aligned}
$$

where $\xi$ and $\xi_{p}$ are defined as

$$
\xi=\frac{\mu_{s} T_{s}}{\mu_{l} T_{l}},
$$

and

$$
\xi_{p}=\frac{\mu_{p} T_{p}}{\mu_{l} T_{l}} .
$$

In this formulation Eq. (9) is a key result because it represents the effect of a finite thickness third layer of elastic mass upon a layer guided system of a finite thickness substrate with a finite thickness wave-guiding layer. When the third layer vanishes $(h \rightarrow 0)$, Eq. (9) is the equation that defines the operating point on the dispersion curve for the "bare" device of a substrate with a waveguide layer. Subsequently, considering the third layer to have a finite, but small, thickness gives the perturbation of the operating point due to sensed mass and so enables the shift in velocity to be calculated. In the case of liquid or polymer loading, it is necessary to identify changes in the previous formulation so as to allow the perturbation to have an arbitrary thickness of liquid or polymer. The generalization to a liquid or polymer will necessarily introduce an attenuation of the wave in addition to the velocity shift.

\section{B. Viscoelasticity and the Maxwell model}

In this section we show that even when a viscoelastic layer is introduced it is possible to retain the majority of the equations used in the previous section in developing the model of a three-layer system with overlayers composed of elastic mass. To incorporate viscoelasticity, first consider the Navier-Stokes equation for a liquid under the assumptions that the liquid is viscous and incompressible and that the pressure gradient can be ignored

$$
\frac{\partial \underline{v}_{f}}{\partial t}=\frac{\eta_{f}}{\rho_{f}} \underline{\nabla}^{2} \underline{\nu}_{f},
$$

where $\nu_{f}$ is the fluid velocity and $\eta_{f}$ is the viscosity of the fluid. Taking a time dependence of $e^{j \omega t}$ the velocity can be rewritten in terms of displacements as

$$
\rho_{f} \frac{\partial^{2} \underline{u}_{f}}{\partial t^{2}}=j \omega \eta_{f} \underline{\nabla}^{2} \underline{u}_{f} .
$$

Comparing Eq. (13) with Eq. (1), we have the same equation except for the replacement of the shear modulus $\mu$ by the liquid factor $j \omega \eta_{f}$. In a similar manner the stress condition in transforming from a solid to the liquid is altered only by this same replacement. Thus, replacement of the shear modulus $\mu$ by a complex shear modulus $G_{f}$, with limits of $\mu$ when the material is solid-like and $j \omega \eta_{f}$ when the material is liquid-like provides one possible model for acoustic wave response with a viscoelastic layer.

In the Maxwell model of viscoelasticity the shear stress and rate of strain can be viewed as a spring and dashpot model. The total rate of strain contains an elastic part and a viscous part and a relaxation time $\tau=\eta_{f} / \mu$ can be introduced. The shear modulus becomes

$$
G_{f}=\frac{j \omega \eta_{f}}{1+j \omega \tau}
$$

so that the limit $\omega \tau \rightarrow \infty$ gives the solid limit $\left(G_{f} \longrightarrow \mu\right)$ and the limit $\omega \tau \rightarrow 0$ gives the liquid limit $\left(G_{f} \longrightarrow j \omega \eta_{f}\right)$. Thus, 
introducing viscoelasticity into the equations of the previous section amounts to the replacement $\mu \rightarrow G_{f}$ in Eqs. (1)-(11).

\section{The shear wave penetration depth}

In the Newtonian liquid limit $\omega \tau \rightarrow 0$, we would expect the shear wave viscous penetration depth defined by $\delta$ $=\left(2 \eta_{f} / \omega \rho_{f}\right)^{1 / 2}$ to be an important length scale determining whether the layer thickness should be regarded as large or small. It is therefore useful to consider the relationship between the wave vector for a layer and the fluid velocity defined using the Maxwell model of viscoelasticity. The wave vector for a fluid layer [Eq. (6) or (7)] can be written

$$
T_{f}^{2}=\omega^{2}\left(\frac{1}{\nu_{f}^{2}}-\frac{1}{\nu^{2}}\right)
$$

where

$$
\nu_{f}^{2}=\frac{G_{f}}{\rho_{f}}=\frac{j \omega \eta_{f}}{\rho_{f}(1+j \omega \tau)} .
$$

Using Eq. (16) in Eq. (15) and recalling the definition of the penetration depth, we can write

$$
T_{f}^{2}=\left(\frac{-2 j(1+j \omega \tau)}{\delta^{2}}-\frac{\omega^{2}}{\nu^{2}}\right)=\frac{-2 j}{\alpha^{2}},
$$

where $\alpha$ has been defined by

$$
\alpha=\frac{\delta}{\sqrt{1+j \omega \tau-\frac{j \delta^{2} \omega^{2}}{2 \nu^{2}}}}=\frac{\delta}{\sqrt{1+j \omega \tau-\frac{j \delta^{2} k^{2}}{2}}} .
$$

In the limit $\delta k \ll 1$ (i.e., the penetration depth is much smaller than $\lambda)$ the fluid wave vector becomes $T_{f}=(-1$ $+j)(1+j \omega \tau)^{1 / 2} / \delta$ and is independent of the wave speed $\nu$. Then in the limit of a Newtonian liquid $\omega \tau \rightarrow 0$, the solution for the fluid displacement is a damped oscillation in the $x_{3}$ direction, whereas for a solid with $\omega \tau \rightarrow \infty$ the wave vector $T_{f}$ becomes real and the solution for the fluid displacement is an oscillation in the $x_{3}$ direction without damping.

\section{VISCOELASTIC GUIDING LAYER}

The first generalization of the previously published model of mass sensitivity ${ }^{21}$ is to allow the waveguiding layer itself to become viscoelastic. This viscoelasticity means that the "bare" device of the substrate and the waveguiding layer has a complex dispersion curve with a wave velocity that has both real and imaginary parts indicating that both a velocity shift and attenuation occur due to the waveguiding layer. The decay in the displacement amplitude of the substrate for a propagation of the wave over a length $L$ is given by Eq. (2) as $\exp \left(\operatorname{Im} k_{1} L\right)$, where Im indicates that the imaginary part of $k_{1}=\omega / \nu$ should be taken. The insertion loss (IL) in decibels per meter propagation length is then given by

$$
\mathrm{IL}=-20\left(\log _{10} e\right) \operatorname{Im}\left[\frac{\omega}{\nu}\right] .
$$

In Eq. (19), the sign convention adopted for the insertion loss is that a larger positive value indicates a weaker transmission of the wave. When considering small changes from an operating point, the inverse wave speed can be expanded about the unperturbed wave speed $v_{0}$ as $1 / \nu \approx 1\left(/ \nu_{0}\right)\left(1-\Delta \nu / \nu_{0}\right)$ and the change in insertion loss can then be evaluated from the change in the complex velocity.

\section{A. Perturbation from a bare substrate}

In this section we consider a bare substrate composed of elastic mass supporting either a SH-SAW or a SH-APM. These two situations correspond to solutions of Eq. (9) with $h=0$ and either $\xi^{0}=0$ or $\tanh \left(T_{s}^{0} w\right)=0$ with $T_{s} \neq 0$, respectively; the superscript zero implies the unperturbed solution for a bare substrate with no viscoelastic layer [i.e., Eq. (9) with both $d=0$ and $h=0$ ]. If a thin layer of elastic mass with $v_{l}<v_{s}$ is deposited, the SH-SAW becomes a Love wave while the SH-APM mode becomes a layer-guided SH-APM mode. In the case of the SH-APM, $T_{s}^{0}$ is purely imaginary so that the $\tanh ()$ function becomes a $\tan ()$ function and $T_{s}^{0} w$ $=j m \pi$, with $m=1,2,3, \ldots$. To develop perturbation solutions for the effect of a viscoelastic layer of thickness $d$ on the bare substrate, we first rewrite Eq. (9) using the perturbed velocity $v=v_{0}+\Delta v$

$$
\tan \left(T_{f}^{0} d\right)=\left(\xi^{0}+\Delta \xi\right)\left[\frac{\tanh \left(T_{s}^{0} w\right)+\tanh \left(\Delta T_{s} w\right)}{1+\tanh \left(T_{s}^{0} w\right) \tanh \left(\Delta T_{s} w\right)}\right]
$$

Continuation of the perturbation solution now depends upon whether $\xi^{0}=0$ or $\tanh \left(T_{s}^{0} w\right)=0$. In the former case, care must be taken not to divide using $T_{s}^{0}$ because $\xi^{0}=0$ implies $T_{s}^{0}=0$ (i.e., $T_{s}$ itself is of order $\Delta T_{s}$ ). Since we are interested in the limit of the viscoelastic layer becoming an infinitely deep Newtonian liquid, we do not assume that $d$ is necessarily small.

\section{SH-SAW perturbation}

The perturbation of the SH-SAW uses an expansion about $\xi^{0}=0$ which implies $T_{s}^{0}=0$ (i.e., $v_{0}=v_{s}$ ). In this case, Eq. (20) simplifies to

$$
\tan \left(T_{f}^{0} d\right) \approx \Delta \xi^{0} \tanh \left(\Delta T_{s} w\right)
$$

The perturbation in the substrate wave vector must be handled carefully because of $T_{s}^{0}=0$, but doing so and grouping together the terms involving the perturbation of the velocity gives

$$
\begin{aligned}
& \left(\frac{\Delta \nu}{\nu_{s}}\right) \tanh ^{2}\left(\frac{w \omega}{\nu_{s}} \sqrt{\frac{-2 \Delta \nu}{\nu_{s}}}\right) \\
& \approx-\frac{1}{2}\left(\frac{G_{f} T_{f}^{0} \nu_{s}}{\mu_{s} \omega}\right)^{2} \tan ^{2}\left(T_{f}^{0} d\right)
\end{aligned}
$$

and using Eqs. (14), (17), and (18) for the Maxwell model of viscoelasticity gives 


$$
\begin{gathered}
\left(\frac{\Delta \nu}{\nu_{s}}\right) \tanh ^{2}\left(\frac{w \omega}{\nu_{s}} \sqrt{\frac{-2 \Delta \nu}{\nu_{s}}}\right) \\
\approx-\frac{1}{2}\left\{\left(\frac{2 \eta_{f} d}{\rho_{s} \nu_{s}(1+j \omega \tau) \alpha_{0}^{2}}\right)\right. \\
\left.\times\left[\frac{\tan \left(\frac{\sqrt{-2 j} d}{\alpha_{0}}\right)}{\left(\frac{\sqrt{-2 j} d}{\alpha_{0}}\right)}\right]\right\}^{2},
\end{gathered}
$$

where $\alpha_{0}$ is Eq. (18) with $\nu=\nu_{s}$. In the limit of an infinitely thick substrate and assuming the real part of $\Delta \nu$ is negative, the $\tanh ()$ term on the left-hand side of Eq. (23) tends to unity. Equations (22) and (23) provide the equations necessary for calculating the velocity shifts and damping of a pure SH-SAW due to a layer of elastic mass, Newtonian liquid, or viscoelastic layer of arbitrary thickness.

Solid and Newtonian liquid limits for SH-SAW. The limit of a thin layer uses $d \rightarrow 0$, so that Eq. (23) gives

$$
\begin{aligned}
& \left(\frac{\Delta \nu}{\nu_{s}}\right) \tanh ^{2}\left(\frac{w \omega}{\nu_{s}} \sqrt{\frac{-2 \Delta \nu}{\nu_{s}}}\right) \\
& \quad \approx-\frac{1}{2}\left(\frac{2 \eta_{f} d}{\rho_{s} \nu_{s}(1+j \omega \tau) \alpha_{0}^{2}}\right)^{2}
\end{aligned}
$$

and taking the solid limit using both $\omega \tau \rightarrow \infty$ and $\delta^{2} \omega^{2} / 2 \nu_{s}^{2}$ $\rightarrow \infty$, this reduces to

$$
\left(\frac{\Delta \nu}{\nu_{s}}\right) \tanh ^{2}\left(\frac{w \omega}{\nu_{s}} \sqrt{\frac{-2 \Delta \nu}{\nu_{s}}}\right) \approx-\frac{1}{2}\left[\frac{\mu_{f}}{\mu_{s}}\left(\frac{\nu_{s}^{2}}{\nu_{f}^{2}}-1\right) \frac{\omega d}{\nu_{s}}\right]^{2},
$$

which is the same as Eq. (33) in Ref. 20. The limit $\omega \tau \rightarrow \infty$ and $\delta^{2} \omega^{2} / 2 v_{s}^{2}=\delta^{2} k_{s}{ }^{2} / 2 \rightarrow 0$ is equivalent to taking the limit $v_{s} \gg v_{f}$ in Eq. (25), and would be correct for a solid layer used as a waveguide in a Love wave device. The limit of an infinitely thick layer of Newtonian liquid uses $d \rightarrow \infty$ with $\omega \tau \rightarrow 0$. In this limit, the $\tan ()$ function on the right-hand side of Eq. (23) tends to $-j$ and therefore

$$
\left(\frac{\Delta \nu}{\nu_{s}}\right) \tanh ^{2}\left(\frac{w \omega}{\nu_{s}} \sqrt{\frac{-2 \Delta \nu}{\nu_{s}}}\right) \approx \frac{j \omega \eta_{f} \rho_{f}}{2 \mu_{s} \rho_{s}}\left(1-\frac{j \delta^{2} k_{s}^{2}}{2}\right),
$$

where $k_{s}=\omega / v_{s}$. In the further limit $\delta^{2 \omega 2} / 2_{s}^{2} \rightarrow 0$, the velocity perturbation [Eq. (26)] is purely imaginary and the effect is to create a damping, but no phase velocity shift. From Eq. (19) and in the limit of $w \rightarrow \infty$, the insertion loss is proportional to the square of the frequency times the viscositydensity product; this can be contrasted with the square root of the viscosity-density product expected for QCM sensors. The limit of an infinitely thick viscoelastic layer can also be obtained from Eq. (23) and would result in Eq. (26) with the replacement

$$
\left(1-\frac{j \delta^{2} k_{s}^{2}}{2}\right) \rightarrow \frac{1+j \omega \tau-\frac{j \delta^{2} k_{s}^{2}}{2}}{(1+j \omega \tau)^{2}}
$$

\section{SH-APM $(m>0)$ perturbation}

In the previous section we considered the perturbation of a SH-SAW using the unperturbed condition $\xi^{0}=0$ which implies $T_{s}^{0}=0$ (i.e., $\nu_{0} \neq \nu_{s}$ ). In the plate mode case we take $T_{s}^{0} \neq 0$ (i.e., $\nu_{o} \neq \nu_{s}$ ) and purely imaginary so that the $\tanh ($ ) function becomes a $\tan ()$ function. The (nontrivial) zeros of this $\tan ()$ function are then our unperturbed solutions and correspond to $T_{s}^{0} w=j m \pi$, with $m=1,2,3, \ldots$. The $m=0$ solution belongs to the SH-SAW case and is not a plate mode solution in the sense that any added elastic mass with $\nu_{l}$ $<\nu_{s}$, no matter how small, converts $T_{s}$ from imaginary to real. For the perturbation of a bare substrate supporting a SH-APM, Eq. (20) reduces to

$$
\tan \left(T_{f}^{0} d\right)=\xi^{o} \tanh \left(\Delta T_{s} w\right)
$$

and performing the perturbation about $T_{s}^{0} \neq 0$ and using Eq. (17) gives

$$
\left(\frac{\Delta \nu}{\nu_{m}}\right) \approx\left(\frac{2 j d v_{m}^{2} G_{f}}{\mu_{s} w \omega^{2} \alpha_{m}^{2}}\right)\left[\frac{\tan \left(\frac{\sqrt{-2 j} d}{\alpha_{m}}\right)}{\left(\frac{\sqrt{-2 j} d}{\alpha_{m}}\right)}\right],
$$

where $\alpha_{m}$ is Eq. (18) with $\nu=\nu_{m}$. In Eq. (29) we have used $\nu_{m}$ to indicate that the unperturbed speed is the plate mode speed and used $\nu_{m}>\nu_{s}$ to convert the $\tanh ()$ function into a $\tan ()$ function. Equation (29) provides the equation necessary for calculating the velocity shifts and damping of a pure SH-APM due to a layer of elastic mass, Newtonian liquid, or viscoelastic layer of arbitrary thickness.

Solid and Newtonian liquid Limits for SH-APM. The thin layer limit $d \rightarrow 0$, is simply the prefactor in Eq. (29)

$$
\left(\frac{\Delta \nu}{\nu_{m}}\right) \approx\left(\frac{-2 \eta_{f} d \nu_{m}^{2}}{\mu_{s} w \omega(1+j \omega \tau) \alpha_{m}^{2}}\right)
$$

and in the solid limit of $\omega \tau \rightarrow \infty$ and $\delta^{2} \omega^{2} / 2 v_{s}^{2} \rightarrow \infty$ this further reduces to

$$
\left(\frac{\Delta \nu}{\nu_{m}}\right) \approx \frac{-\mu_{f}}{\mu_{s}}\left(\frac{\nu_{m}^{2}}{\nu_{f}^{2}}-1\right) \frac{d}{w},
$$

which is the same as Eq. (42) in Ref. 20. It should be noted that since $T_{s}^{0} w=j m \pi$ the substrate thickness $w$ is proportional to $1 / \omega$, i.e.,

$$
w \omega=\frac{m \pi \nu_{m}}{\sqrt{\frac{\nu_{m}^{2}}{\nu_{s}^{2}}-1}}
$$

and Eq. (31) therefore predicts a fractional shift in phase 
velocity proportional to the mode frequency. Also the factor $\mu_{f} d=\nu_{f}^{2} \rho_{f} d$ and so involves the combination of density and thickness, thus giving the mass per unit area. Equation (31) is therefore similar to the Sauerbrey equation familiar from QCM sensors in that it predicts a fractional shift in phase speed proportional to the frequency multiplied by the mass per unit area. The limit $\omega \tau \rightarrow \infty$ and $\delta^{2} \omega^{2} / 2 \nu_{s}^{2} \rightarrow 0$ is equivalent to taking the limit $\nu_{m} \gg \nu_{f}$ in Eq. (29).

The limit of an infinitely thick viscoelastic layer uses $d$ $\rightarrow \infty$ in the $\tan$ () function on the right-hand side of Eq. (29). In this limit, the $\tan ()$ function tends to $j$ and so

$$
\left(\frac{\Delta \nu}{\nu_{m}}\right) \approx \frac{\sqrt{-2 j} \eta_{f} \nu_{m}^{2}}{\nu_{s}^{2} \rho_{s} w \omega(1+j \omega \tau) \alpha_{m}}
$$

and in the limit $\delta \rightarrow 0$ we find

$$
\left(\frac{\Delta \nu}{\nu_{m}}\right) \approx \frac{1}{\sqrt{2} w \omega}\left(\frac{\nu_{m}^{2} \sqrt{\rho_{f} \eta_{f} \omega}}{\nu_{s}^{2} \rho_{s}}\right)\left[-F_{-}(\omega \tau)+j F_{+}(\omega \tau)\right],
$$

where the $F_{ \pm}(\omega \tau)$ functions are defined by

$$
F_{ \pm}(\omega \tau)=\left(\frac{\sqrt{1+(\omega \tau)^{2}} \pm \omega \tau}{1+(\omega \tau)^{2}}\right)^{1 / 2} .
$$

There is a strong relationship between Eq. (34) and the results quoted by Martin et al. ${ }^{22}$ and Ricco and Martin ${ }^{23}$ (1990) with similar dependencies on various physical factors including the $F_{ \pm}(\omega \tau)$ functions. However, there is a difference in the prefactor with our $\nu_{s}^{2} / 2 \nu_{m}$ replacing a mode group velocity $\nu_{g m}$ factor in the Martin et al. formula; ${ }^{22}$ this difference is detailed in Appendix A.

\section{B. General perturbation of a viscoelastic layer-guided wave}

When a SH-SAW device is coated with an elastic mass layer with a shear acoustic speed less than that of the substrate, the wave becomes a Love wave with a speed intermediate between that of the layer and the substrate. Similarly, we have previously shown that coating a SH-APM device with an elastic mass layer with a shear acoustic speed less than that of the substrate creates a layer-guided SH-APM with a wave speed intermediate between that of the original mode and the next lower SH-APM mode. In both the Love wave and layer-guided SH-APM cases, the use of a coating of elastic mass results in a change in the wave speed, but does not cause any propagation loss. For use as sensors, the benefit of the coating, also described as a waveguiding layer, is that an enhanced sensitivity to mass deposition can be obtained. If the waveguiding layer were a viscoelastic material we would expect an insertion loss to be introduced in addition to a shift in the phase speed. The formalism of Sec. II can be used to determine the general effect of depositing a polymer layer (or a mass layer or immersing the device in a liquid) on a layer-guided wave device. In this section we consider both the waveguiding layer and the third, perturbing, layer to be viscoelastic; the thickness of the third layer is kept arbitrary and is not assumed small. The device response to mass or liquid loading can then be obtained by taking appropriate limits.

The unperturbed system of a substrate with a viscoelastic waveguide layer is defined by Eq. (9) with $h=0$

$$
\tan \left(T_{l}^{0} d\right)=\xi^{o} \tanh \left(T_{s}^{0} w\right),
$$

where $\xi^{0}$ has been defined using Eq. (10), but with viscoelastic parameters (i.e., $\mu_{l} \rightarrow G_{l}$ ). Similarly, the $T_{l}^{0}$ includes the viscoelasticity in Eq. (6) via the use of equations of the form given in Eqs. (15)-(18). For simplicity the substrate is assumed to be composed of elastic mass. We also assume that the unperturbed velocity $v_{0}$ does not equal $v_{s}$, so that we are considering an operating point located away from the start of a mode on the dispersion curve for the system of the substrate and guiding layer. The perturbation due to the third layer, which is assumed finite and viscoelastic, is then given by Eq. (9) and is symbolically similar to the derivation of Eq. (24) in Ref. 21, except we keep the third layer thickness finite and the shear moduli and, hence, velocities of the waveguide and perturbing layers are allowed to be complex. Thus, using a subscript $f$ to represent quantities for the third, perturbing, layer of thickness $h$, the complex perturbation is

$$
\begin{aligned}
\frac{\Delta \nu}{\nu_{0}} \approx & g\left(\omega, \nu_{0}, \nu_{s}, G_{l}, \nu_{l}, w, d\right)\left(1-\frac{\nu_{f}^{2}}{\nu_{0}^{2}}\right) \\
& \times\left(\frac{\tan \left(T_{f}^{0} h\right)}{T_{f}^{0} h}\right) \rho_{f} h,
\end{aligned}
$$

where the function $g$ has been defined as

$$
g=\frac{\frac{-\omega^{2}}{G_{l} T_{l}^{0}}\left[1+\tan ^{2}\left(T_{l}^{0} d\right)\right]}{\left\{\frac{T_{l}^{0} d}{\left(\frac{\nu_{0}^{2}}{\nu_{l}^{2}}-1\right)}\left[1+\tan ^{2}\left(T_{l}^{0} d\right)\right]+\frac{\xi^{0} T_{s}^{0} w}{\left(1-\frac{\nu_{0}^{2}}{\nu_{s}^{2}}\right)}\left[1-\tanh ^{2}\left(T_{s}^{0} w\right)\right]+\tan \left(T_{l}^{0} d\right)\left[\frac{1}{\left(\frac{\nu_{0}^{2}}{\nu_{l}^{2}}-1\right)}+\frac{1}{\left(1-\frac{\nu_{0}^{2}}{\nu_{s}^{2}}\right)}\right]\right\}} .
$$


The equivalent equation defining $g$ in Ref. 21 [i.e., Eq. (25)] contains several typographical errors. Noting that in the case of elastic mass, $G_{p} / \nu_{p}^{2} \rightarrow \rho_{p}$ and $G_{l} \rightarrow \rho_{l} \nu_{l}^{2}$, formally Eq. (37) appears to be the same as in the case of elastic mass derived in Ref. 21 except for the additional $\tan x / x$ type multiplicative factor arising from maintaining a finite thickness, rather than an infinitesimally thin, third layer. If the waveguide layer is simply elastic mass then both the function $g$ and the unperturbed speed $v_{0}$ are real and any complex component to $\Delta \nu$ arises purely from the viscoelasticity of the perturbing layer. However, if the waveguide layer is viscoelastic then both $g$ and $\nu_{0}$ may be complex. In this situation, a third layer having a real shear modulus will, from Eq. (37), give rise to a complex velocity shift $\Delta \nu$ [i.e., an insertion loss occurs via Eq. (19)]. Thus, mass deposition onto a device possessing a viscoelastic guiding layer can induce an insertion loss response in addition to a frequency (or phase) response. In the SH-APM limit of $d=0$ and $\tanh \left(T_{s}^{0} w\right)=0$ with $T_{s} \neq 0$ (i.e., $\left.\nu_{o} \neq \nu_{s}\right), g \rightarrow \nu_{0}^{2} /\left(\nu_{s}^{2} w \rho_{s}\right)$ and Eq. (37) reduces to Eq. (29).

\section{Maxwell model for perturbing layer}

The effects of viscoelasticity in Eq. (37) can be made more evident by rewriting it using Eqs. (16) and (17)

$$
\begin{aligned}
\frac{\Delta \nu}{\nu_{0}} \approx & g\left(\omega, \nu_{0}, \nu_{s}, G_{l}, \nu_{l}, w, d\right)\left(1-\frac{G_{f}}{\rho_{f} \nu_{0}^{2}}\right) \\
& \times\left(\frac{\tan \left(\frac{\sqrt{-2 j} h}{\alpha_{f}^{0}}\right)}{\left(\frac{\sqrt{-2 j} h}{\alpha_{f}^{0}}\right)}\right) \rho_{f} h,
\end{aligned}
$$

where $G_{f}$ is given by Eq. (14) and $\alpha_{f}^{0}$ by Eq. (18). In the Maxwell model the second factor in Eq. (39) can be written using the fluid penetration depth and relaxation time as

$$
\left(1-\frac{G_{f}}{\rho_{f} \nu_{0}^{2}}\right)=\left(1-\frac{j \delta_{f}^{2} k_{0}^{2}}{2\left(1+j \omega \tau_{f}\right)}\right) .
$$

In the limit of a thin viscoelastic layer, $\tan x / x \rightarrow 1$ and the perturbation becomes $\Delta \nu / \nu \propto \rho_{f} h$, which in the solid limit $\left(\omega \tau_{f} \rightarrow \infty\right)$ becomes mass per unit area. In the limit of an infinitely thick viscoelastic liquid, $\tan \left[(-2 j)^{1 / 2} x\right] /\left[(-2 j)^{1 / 2} x\right]$ $\rightarrow j /\left[(-2 j)^{1 / 2} x\right]$ and assuming that $\delta_{f}^{2} \omega^{2} / 2 \nu_{0}^{2} \rightarrow 0$ we obtain the analogous equation to Eq. (34)

$$
\begin{aligned}
\frac{\Delta \nu}{\nu_{0}} \approx & g\left(\omega, \nu_{0}, v_{s}, G_{l}, \nu_{l}, w, d\right)\left(\frac{1}{\sqrt{2} \omega}\right) \sqrt{\rho_{f} \eta_{f} \omega} \\
& \times\left[-F_{-}\left(\omega \tau_{f}\right)+j F_{+}\left(\omega \tau_{f}\right)\right],
\end{aligned}
$$

which reduces to Eq. (34) when the $d \rightarrow 0$ limit for $g$ is used.

\section{Relationship to the slope of the dispersion curve}

Equation (39) shows that the same function $g$ determines the sensitivity toward both mass and liquid perturbing layers since the function depends only on the operating frequency and substrate and waveguide layer properties. Therefore, us- ing Eq. (37) for the thin mass limit and Eq. (41) for the liquid limit, the ratio of the response of a Love wave or layerguided SH-APM mode due to coating by a thin mass layer or due to immersion in a perturbing liquid is

$$
\frac{(\Delta \ln \nu)_{\text {mass }}}{(\Delta \ln \nu)_{\text {liquid }}} \approx \sqrt{2 \omega}\left(1-\frac{\nu_{p}^{2}}{\nu_{0}^{2}}\right) \frac{\Delta m}{\sqrt{\rho_{f} \eta_{f}}},
$$

where $\Delta m=\rho_{m} h$ is the mass per unit area, $\nu_{m}$ is the shear acoustic speed of that mass, and the fractional perturbation in the wave speed has been written as a perturbation in the logarithm of the wave speed. In many senses this is a similar relationship to that between the Sauerbrey response of a QCM and the Kanazawa expression for the liquid response of a QCM. In the QCM case the ratio of the responses would involve a $\sqrt{ } \omega$ and the ratio of the mass per unit area to the square root of the liquid density-viscosity product. For the layer-guided wave it should be noted that the operating point on the dispersion curve for the device (substrate plus guiding layer) determines $\nu_{0}$ and this effectively introduces a frequency dependence, although in many situations $\nu_{m}^{2} \ll \nu_{0}^{2}$. While the frequency dependence of the ratio of the mass and liquid responses is similar to the QCM result, Eq. (38) does not imply that the absolute frequency dependence of the layer-guided system is the same as the QCM frequency response. However, what is particularly important experimentally is that if we can determine the sensitivity function $g$ for any perturbing layer then it is the same function for any other layer. ${ }^{21}$ While we have previously noted the importance of this last observation, our present work shows that its relevance is much wider than previously indicated. The function $g$ can be determined using a thin elastic mass layer, but will then be valid whether the device is used for sensing mass deposited from the vapor phase or for sensing liquid (or polymer) properties. Moreover, the sensitivity function $g$ for a sensor created using a viscoelastic waveguide layer can be related to the slope of the dispersion curve in an identical manner to that previously suggested for a waveguide layer composed of elastic mass. ${ }^{21}$ Thus, by considering a third perturbing layer composed of a thin layer of the same viscoelastic material as used to create the waveguide layer, we can use Eq. (37) to derive

$$
g\left(\omega, \nu_{0}, \nu_{s}, G_{l}, \nu_{l}, w, d\right)=\frac{1}{\rho_{l}\left(1-\nu_{l}^{2} / \nu_{0}^{2}\right)}\left(\frac{d \log _{e} \nu}{d x}\right)_{x=d} .
$$

Defining a new dimensionless variable $z=d f / \nu_{l}^{\infty}$ (i.e., $z$ $=d / \lambda_{l}^{\infty}$ ), where the superscript $\infty$ implies the solid limit (i.e., $\omega \tau \rightarrow \infty$ in the Maxwell model) the perturbation of the complex velocity, Eq. (37), becomes

$\frac{\Delta \nu}{\nu_{0}} \approx\left(\frac{1-\nu_{f}^{2} / \nu_{0}^{2}}{1-\nu_{l}^{2} / \nu_{0}^{2}}\right)\left(\frac{d \log _{e} \nu}{d z}\right)_{z=z_{0}}\left(\frac{\tan \left(T_{f}^{0} h\right)}{T_{f}^{0} h}\right) \frac{\omega \rho_{f} h}{2 \pi \nu_{l}^{\infty} \rho_{l}}$

and in the Maxwell model of viscoelasticity $T_{f}^{0} h$ $=(-2 j)^{1 / 2} h / \alpha_{f}^{0}$. 


\section{Relationship to the Sauerbrey and Kanazawa equations}

Equation (44) is a key result of this work because it strongly emphasizes the formal similarity between the response of Love wave and layer-guided SH-APM devices with that of QCMs. It deserves to be regarded as a generalization of the Sauerbrey equation for QCM response to mass loading and the Kanazawa result for liquid loading to layerguided sensors. The limits of the $\tan x / x$ type function in Eq. (44) provide results for the thin solid film and infinitely deep Newtonian liquid

$$
\left(\frac{\tan \left(T_{f}^{0} h\right)}{T_{f}^{0} h}\right) \rightarrow\left\{\begin{array}{cc}
1 & h \rightarrow 0 \\
\frac{-\sqrt{-2 j}}{2 h\left(1-\nu_{f}^{2} / \nu_{0}^{2}\right)} \sqrt{\frac{2 \eta_{f}}{\omega \rho_{f}}} & h \rightarrow \infty \quad \text { and } \omega \tau \rightarrow 0
\end{array}\right.
$$

According to this equation, subject to two conditions, the fractional change in wave speed of a sensor due to an infinitesimally thin layer of rigidly bound elastic mass is proportional to the frequency multiplied by the mass per unit area (i.e., $\left.\omega \rho_{f} h=\omega \Delta m\right)$ and the response to immersion in an infinitely deep Newtonian liquid is proportional to the square root of frequency multiplied by the square root of the density-viscosity product i.e., $\left(\omega \rho_{f} \eta_{f}\right)^{1 / 2}$. The two conditions that must be fulfilled are that the operating point on the dispersion curve does not change and that $\nu_{f}^{2} \ll \nu_{0}^{2}$. However, the first of these conditions is a strong condition because a general change of frequency without changing the waveguiding layer thickness will necessarily alter the factor in Eq. (44) that involves the derivative of the phase velocity. It is therefore not generally true that the frequency dependence of the $\Delta \nu / \nu_{0}$ response to mass and liquid loading is $\omega$ and $\omega^{1 / 2}$, respectively, although the mass and liquid responses should differ by a factor of $\omega^{1 / 2}$.

\section{MASS DEPOSITION FROM A LIQUID}

The perturbation summarized by Eq. (44) is the result of adding a viscoelastic layer to a Love wave or layer-guided SH-APM device in vacuum. When the added layer is a Newtonian liquid of infinite depth the perturbation is a comparison between the wave velocity for the device when immersed to the wave velocity for the device in vacuum. When the added layer is an infinitesimally thin mass layer, the comparison is again to the wave velocity for the device in vacuum. In sensor work with QCMs it is approximately the case that mass deposition from the liquid phase creates the same perturbation as the same mass deposition, but from the vapor phase. For Love wave and layer-guided SH-APMs mass deposition from a liquid phase introduces a fourth layer and the unperturbed situation corresponds to three layers (Fig. 2). The layer that can be regarded as the perturbation is positioned between the waveguide and the final (fourth) layer. Following the pattern of Sec. III 2 B, it is tempting, but strictly incorrect, to conclude that mass deposition from a viscoelastic liquid phase will be described by Eq. (44) with $h=\Delta h$ representing a mass layer and the unperturbed solution being the immersed Love wave or layer-guided device, i.e.,

$$
\frac{\Delta \nu}{\nu_{0}} \approx\left(\frac{1-\nu_{m}^{2} / \nu_{0}^{2}}{1-\nu_{l}^{2} / \nu_{0}^{2}}\right)\left(\frac{d \log _{e} \nu}{d z}\right)_{z=z_{0}} \frac{\omega \rho_{m} \Delta h}{2 \pi v_{l}^{\infty} \rho_{l}},
$$

where $\nu_{0}$ is a solution of the three-layer equation [Eq. (9)]

$$
\begin{aligned}
\tan \left(T_{l}^{0} d\right)= & \xi_{s l}^{0} \tanh \left(T_{s}^{0} w\right)-\xi_{f l}^{0} \tan \left(T_{f}^{0} b\right) \\
& \times\left\lfloor 1+\xi_{s l}^{0} \tan \left(T_{l}^{0} d\right) \tanh \left(T_{s}^{0} w\right)\right\rfloor .
\end{aligned}
$$

The symbols $\xi_{s l}$ and $\xi_{f l}$ have been defined as

$$
\xi_{s l}=\frac{\mu_{s} T_{s}}{G_{l} T_{l}},
$$

and

$$
\xi_{f l}=\frac{G_{f} T_{f}}{\mu_{l} T_{l}} .
$$

Equation (47) can be rearranged into the form

$$
\tan \left(T_{l}^{0} d\right)=\frac{\xi_{s l}^{0} \tanh \left(T_{s}^{0} w\right)-\xi_{f l}^{0} \tan \left(T_{f}^{0} b\right)}{1+\xi_{s l}^{0} \xi_{f l}^{0} \tan \left(T_{f}^{0} b\right) \tan \left(T_{l}^{0} d\right) \tanh \left(T_{s}^{0} w\right)} .
$$

For a device immersed in an infinitely deep viscoelastic medium the limit $b \rightarrow \infty$ would need to be taken. If Eq. (46) were correct, then the perturbation due to mass deposition from the liquid phase could be related to the mass deposited by experimentally determining the dispersion curve for $v$ in

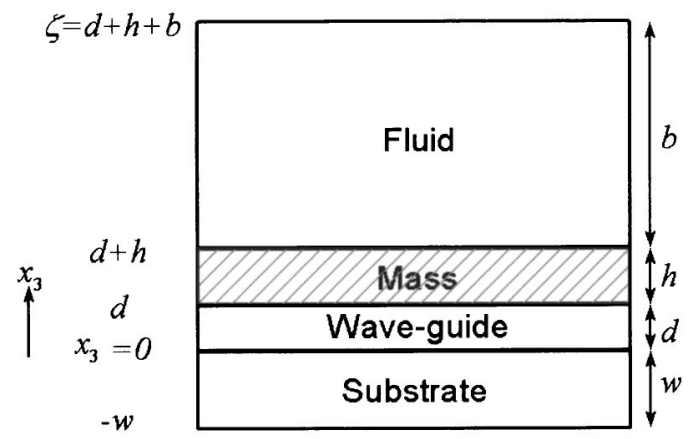

FIG. 2. Layers in the four-layer system. The substrate, guiding-layer, mass layer, and fluid layer are indicated by, $w, l, m$, and $f$, respectively. For mass deposition from the liquid phase the third layer is regarded as the perturbation. 
the liquid as a function of guiding layer thickness in a similar manner to the work reported in Ref. 16. This type of experiment would enable the derivative of the liquid phase dispersion curve to be determined numerically and the sensitivity to be evaluated. These considerations only apply if an equation similar to Eq. (46) can be shown to be valid for mass deposition from a liquid; in the following we provide a rigorous derivation of a slightly modified form of Eq. (46) [see Eq. (52)].

To rigorously investigate the effect of mass deposition from the liquid phase, a full solution in a similar form to Eq. (44) can be obtained from first principles by extending the three-layer model to a four-layer model; the algebra involved is extensive and is briefly outlined in Appendix B. The result for the perturbation in the velocity from the solution to Eq. (47) is

$$
\begin{aligned}
\frac{\Delta \nu}{\nu_{0}} \approx & g_{L}\left(\omega, \nu_{0}, \text { substrate, guiding layer, fluid }\right) \\
& \times\left(1-\frac{\nu_{m}^{2}}{\nu_{0}^{2}}\right)\left[1+\xi_{f m}^{2} \tan ^{2}\left(T_{f}^{0} b\right)\right] \rho_{m} \Delta h,
\end{aligned}
$$

where the function $g_{L}$ depends on the substrate, guidinglayer and viscoelastic fluid properties, and the operating point and frequency. In a manner similar to the derivation of Eq. (43), we can imagine the perturbation resulting from the deposition of a thin mass layer that has identical properties to the waveguiding layer, and this allows us to relate the function $g_{L}$ to the slope of the liquid phase device dispersion curve

$$
\begin{aligned}
\frac{\Delta \nu}{\nu_{0}} \approx & {\left[\frac{1+\xi_{f m}^{0}{ }^{2} \tan ^{2}\left(T_{f}^{0} b\right)}{1+\xi_{f l}^{02} \tan ^{2}\left(T_{f}^{0} b\right)}\right]\left(\frac{1-\nu_{m}^{2} / \nu_{0}^{2}}{1-\nu_{l}^{2} / \nu_{0}^{2}}\right) } \\
& \times\left(\frac{d \log _{e} \nu}{d z}\right)_{z=z_{0}} \frac{\omega \rho_{m} \Delta h}{2 \pi \nu_{l}^{\infty} \rho_{l}},
\end{aligned}
$$

which is similar to Eq. (46) apart from the first factor; $\xi_{f m}$ has been defined in the same manner as Eq. (49). For an infinitely deep viscoelastic liquid assuming a Maxwell model and that the $\delta^{2} k_{0}^{2} / 2$ term in $T_{f}^{0}$ can be neglected, the factors in Eqs. (47), (51), and (52) involving $G_{f} T_{f}^{0}$ become

$G_{f} T_{f}^{0} \tan \left(T_{f}^{0} b\right) \rightarrow \frac{-\omega \sqrt{\rho_{f} \eta_{f} \omega}}{\sqrt{2}}\left[-F_{-}(\omega \tau)+j F_{+}(\omega \tau)\right]$.

In the limit of a Newtonian liquid $\omega \tau \rightarrow 0$, so that $F_{-} \rightarrow 0$ and $F_{+} \rightarrow 1$.

When considering acoustically thin layers of mass deposited onto a QCM sensor operating in the liquid phase it is often assumed that the total shift in velocity is additive. The total velocity shift is viewed as the sum of the shifts that would be obtained for the QCM immersed in the liquid added to the shift that would be obtained if the mass were deposited from the gas phase. The accuracy of this assumption for Love wave and layer-guided SH-APM sensors can be assessed by comparing Eq. (46) with Eq. (52) and defining two mass sensitivity functions

$$
\begin{aligned}
S_{\rho_{f}}^{\mathrm{vacuum}} \equiv & \lim _{h \rightarrow 0} \frac{1}{\rho_{f} h} \operatorname{Re}\left(\frac{\Delta v}{v_{0}}\right)^{\text {vacuum }} \\
= & \operatorname{Re}\left[\left(\frac{1-\nu_{f}^{2} / \nu_{0}^{2}}{1-\nu_{l}^{2} / \nu_{0}^{2}}\right)^{\text {vacuum }}\right. \\
& \left.\times\left(\frac{d \log _{e} v}{d z}\right)_{z=z_{0}}^{\text {vacuum }} \frac{\omega}{2 \pi \nu_{l}^{\infty} \rho_{l}}\right]
\end{aligned}
$$

and

$$
\begin{aligned}
& S_{\rho_{m}}^{\text {fluid }} \equiv \lim _{h \rightarrow 0} \frac{1}{\rho_{m} h} \operatorname{Re}\left(\frac{\Delta \nu}{\nu_{0}}\right)^{\text {fluid }} \\
&=\operatorname{Re}\left[\left(\frac{1+\xi_{f m}^{0}{ }^{2} \tan ^{2}\left(T_{f}^{0} b\right)}{1+\xi_{f l}^{0} \tan ^{2}\left(T_{f}^{0} b\right)}\right){ }^{\text {fluid }}\right. \\
&\left.\times\left(\frac{1-\nu_{m}^{2} / \nu_{0}^{2}}{1-\nu_{l}^{2} / \nu_{0}^{2}}\right)^{\text {fluid }}\left(\frac{d \log _{e} \nu}{d z}\right)_{z=z_{0}}^{\text {fluid }} \frac{\omega}{2 \pi \nu_{l}^{\infty} \rho_{l}}\right] .
\end{aligned}
$$

The superscripted words vacuum and fluid have been used as reminders that the unperturbed reference situations correspond to a device either in vacuum or immersed in a fluid. In order for the additive assumption used in QCM sensor work to also be valid for the layer-guided sensors, a number of conditions need to be satisfied. First, the first factor in Eq. (55) has to be approximately unity. Second, the terms in the second factor in Eq. (55) need to be approximately equal either because the two unperturbed operating point velocities $\nu_{0}$ are close or because $\nu_{m}^{2} / \nu_{0}^{2} \ll 1$ and $\nu_{1}^{2} / \nu_{0}^{2} \ll 1$; the unperturbed $\nu_{o} \mathrm{~s}$ are different in Eqs. (54) and (55) because one is relative to the dispersion curve for the device in vacuum and the other for the device in the fluid. Third and finally, the slopes of the phase speed curves at the operating point should be approximately equal. For Love wave and layerguided SH-APM sensors operated at maximum phase speed sensitivity the second two assumptions may not be true because the maximum phase speed sensitivity will necessarily correspond to the point of steepest slope on the phase speed curve.

\section{NUMERICAL RESULTS AND DISCUSSION FOR LOVE WAVES}

The equations developed in the previous sections are comprehensive and cover many types of sensor situations. The effects of elastic solids, Newtonian liquids, and viscoelastic liquids on SH-SAW and SH-APM sensors are described by Eq. (36). This equation also gives the dispersion curves for both Love waves and layer-guided SH-APMs when the guiding layer is viscoelastic and the dispersion curves can be used to evaluate sensor response via Eq. (44). Equation (47) describes the dispersion curve for a Love wave or layer-guided SH-APM sensor immersed in liquid and this dispersion curve is relevant for the evaluation of sensor response to mass deposited from the liquid phase [Eq. (52)]. While the analytical equations cover a wide range of sensor situations, it is a substantial task to provide comprehensive 
numerical calculations for all these situations. Therefore, in this section we focus solely on the effect of viscoelasticity in the guiding layer on the dispersion curve and the sensor response of a Love wave device.

\section{A. Numerical approach}

To understand the effect of the viscoelasticity of the guiding layer on Love waves it is necessary to numerically compute the dispersion curve Eq. (36) for the complex velocity. The insertion loss can then be calculated from the imaginary part of the inverse wave velocity. The general problem of the substrate plus a viscoelastic layer has three intrinsic scales related to the frequency. Imagining the substrate to be infinitely thick $(w \rightarrow \infty)$ and the guiding layer to be perfectly elastic mass $(\omega \tau \rightarrow \infty)$, the guiding layer thickness becomes a natural intrinsic length scale. By defining the combination $\lambda_{l}=\nu_{l} / f$ a dimensionless combination $d / \lambda_{l}$ can be formed and the velocity is a real value determined by a function of $d / \lambda_{l}$ with no other dependence on frequency. However, if the thickness of the substrate is retained as finite, then another combination $\lambda_{s}=\nu_{s} / f$ becomes possible and the velocity then depends on the frequency in a more complicated manner, although it remains real; a natural dimensionless combination is $w / \lambda_{s}$. When the perfectly elastic mass limit is relaxed so that $\omega \tau$ becomes a natural dimensionless combination, the frequency dependence of the wave speed becomes more complicated and, in addition, the speed becomes complex indicating that an insertion loss also occurs.

Considering a finite substrate composed of elastic mass overlayed by a finite thickness Maxwellian viscoelastic layer there are five material parameters $\left(\rho_{s}, \nu_{s}, \rho_{l}, \nu_{l}^{\infty}, \omega \tau\right)$ and three operating parameters $(w, d, f)$ where $\nu_{l}^{\infty}=\nu_{l}(\omega \tau \rightarrow \infty)$ and the product $\omega \tau$ is treated as a single parameter. Using this parameter set the layer penetration depth is given by $\delta_{l}$ $=\left(2 \eta_{l} / \omega \rho_{l}\right)^{1 / 2}$, the layer speed is $\nu_{l}=\nu_{l}^{\infty}[j \omega \tau /(1$ $+j \omega \tau)]^{1 / 2}$, the layer shear modulus by $G_{l}=j \rho_{l} \nu_{l}^{\infty 2} \omega \tau /(1$ $+j \omega \tau)$, and the viscosity of the layer is given by $\eta_{l}$ $=G_{l}(\omega \tau \rightarrow \infty) \tau$. In analogy to the dispersion curve for a Love wave device on an infinite thickness substrate and with an elastic mass guiding layer, we define a parameter $z$ $=d f / \nu_{l}^{\infty}$. To rewrite Eq. (36) into a form suitable for numerical work, we define a new variable $x=T_{l}^{0} d$ and a complex function $\beta=2 \pi \nu_{l}^{\infty}\left(1-\nu_{l}^{2} / \nu_{s}^{2}\right)^{1 / 2} z / \nu_{l}$. Equation (36) can then be written in either of the two equivalent forms

$$
\tan x=\left(\frac{\mu_{s}}{G_{l}}\right)\left(\frac{\beta}{x}\right) \sqrt{1-\left(\frac{x}{\beta}\right)^{2}} \tanh \left[\frac{\beta w}{d} \sqrt{1-\left(\frac{x}{\beta}\right)^{2}}\right],
$$

or

$$
\tan x=-\left(\frac{\mu_{s}}{G_{l}}\right)\left(\frac{\beta}{x}\right) \sqrt{\left(\frac{x}{\beta}\right)^{2}-1} \tan \left[\frac{\beta w}{d} \sqrt{\left(\frac{x}{\beta}\right)^{2}-1}\right] .
$$

The numerical problem is to compute the complex value of $x$ that is a solution to Eq. (57) [and hence Eq. (56)] given a particular set of material and operating parameters. The complex velocity is then found from

$$
\nu=\frac{\nu_{l}}{\sqrt{1-\left(\frac{x \nu_{l}}{2 \pi z \nu_{l}^{\infty}}\right)^{2}}} .
$$

To understand the numerical problem, we can first consider the limit $\omega \tau \rightarrow \infty$, which reduces $x$ and $\nu$ to being real numbers. For a given parameter set, the solution for $x$ can either lie in the range 0 to $\beta$ or it can be larger than $\beta$, but smaller than $2 \pi\left(1-\nu_{l}^{\infty} / \nu_{s}^{2}\right)^{1 / 2} z$. In the former case, Eq. (56) indicates that the solutions for $x$ will correspond to the intersection of the $\tan x$ with the tanh curve. If $\beta<\pi$ there will be a single solution corresponding to the first Love wave mode, since $x<\beta$ necessarily means from Eq. (58) that $\nu<\nu_{s}$. Each time $\beta$ increases by $\pi$ an additional solution, corresponding to a higher mode Love wave, becomes possible; the number of Love wave modes is given by $1+$ the integer part of $\beta / \pi$. The start of each Love wave mode, labeled by an integer $n$, corresponds to $T_{l}^{0} d=n \pi$ and $\xi^{o}=0$ in Eq. (36) so that $\nu=\nu_{s}$. In the latter case, when $x>\beta$, Eq. (57) is a more suitable form for understanding the equation defining $x$. The problem then corresponds to finding the intersections between the two tan functions, which can have widely differing periods in $x$. These solutions each have $\nu>\nu_{s}$ and correspond to layer-guided SH-APMs. More traditional, nonlayer guided SH-APMs occur when $d=0$ and $T_{s}^{0} d=j m \pi$ and this corresponds to mode speeds $\nu_{m}$ given by

$$
\nu_{m}=\frac{\nu_{s}}{\sqrt{1-\left(\frac{m \pi \nu_{s}}{w \omega}\right)^{2}}} .
$$

Equation (59) can also describe the layer-guided plate mode speeds at the start of each Love wave branch, defined by $T_{l}^{0} d_{n m}=n \pi$. The specific guiding layer thicknesses $d_{n m}$ at which $\nu=\nu_{m}$ is satisfied are given by

$$
d_{n m}=\frac{n \nu_{l} / f}{2 \sqrt{1-\left(\frac{\nu_{l}}{\nu_{s}}\right)^{2}\left[1-\left(\frac{m \nu_{s}}{2 f w}\right)^{2}\right]}} .
$$

Thus $d_{n m}$ can be described as the guiding layer thickness at which the Love waves $(m=0)$ and their associated plate modes $(m \neq 0)$ begin and the wave speeds at the start of these modes are given by $\nu_{m}$.

\section{B. Numerical results for phase speed and insertion loss}

The first effect of allowing the guiding layer to become viscoelastic is that Eq. (57) and its solution $x$ both become complex. Analytically, we can still imagine a set of Love wave and associated layer-guided SH-APM modes with the starts of the modes at values $\left(d_{n m}, \nu_{m}\right)$ given by Eqs. (59) and (60). The wave speed at the start of the mode is real while the guiding layer thickness $d_{n m}$ becomes complex, i.e., the mathematically required $d_{n m}$ for $\nu=\nu_{m}$ becomes an unphysical value. In the remainder of this section we simplify 


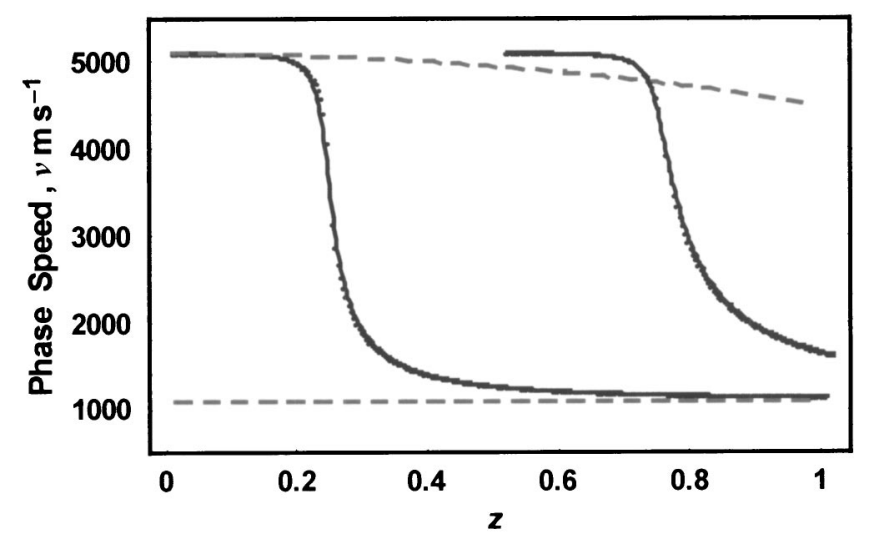

FIG. 3. The real part of the velocity as a function of the normalized guiding layer thickness $z=d f / \nu_{l}(\omega \tau \rightarrow \infty)$ for the first two Love wave modes in a system of a finite thickness substrate with a Maxwellian viscoelastic guiding layer. The solid curves correspond to $\omega \tau=10^{6}$ and the dotted curves to $\omega \tau=10$; the dashed curves are the limits for $\nu \rightarrow \nu_{s}$ and $\nu \rightarrow \nu_{l}$. The other parameters are $f=100 \mathrm{MHz}, w=500 \mu \mathrm{m}, \rho_{s}=2655 \mathrm{~kg} \mathrm{~m}^{-3}, \nu_{s}=5100 \mathrm{~m}$ $\mathrm{s}^{-1}, \rho_{l}=1000 \mathrm{~kg} \mathrm{~m}^{-3}$ and $\nu_{l}(\omega \tau \rightarrow \infty)=1100 \mathrm{~m} \mathrm{~s}^{-1}$.

the computational problem by concentrating on the effects of viscoelasticity of the wave-guide layer on the Love wave solutions and do not consider the layer-guided SH-APM branch of the solutions. Our approach is to choose the material parameters $\left(\rho_{s}, \nu_{s}, \rho_{l}, \nu_{l}^{\infty}, \omega \tau\right)$ and the two operating parameters $w$ and $f$, and to then step through a range of guiding layer thickness $d$. At each step Eq. (57) is numerically solved for the complex root $x$ and the velocity $v$ calculated from Eq. (58). The insertion loss is then calculated from the imaginary part of the inverse velocity using Eq. (19).

Figure 3 shows calculations of the real part of $\nu$ using an operating frequency of $100 \mathrm{MHz}$ on a substrate of thickness $500 \mu \mathrm{m}$ with material parameters of $\rho_{s}=2655 \mathrm{~kg} \mathrm{~m}^{-3}$ and $\nu_{s}=5100 \mathrm{~m} \mathrm{~s}^{-1}$, coated by a viscoelastic layer with material parameters of $\rho_{l}=1000 \mathrm{~kg} \mathrm{~m}^{-3}$ and $\nu_{l}(\omega \tau \rightarrow \infty)=1100 \mathrm{~m}$ $\mathrm{s}^{-1}$. The solid curves show the first two Love modes and use a relaxation time for the waveguiding layer satisfying $\omega \tau$ $=10^{6}$, while the dotted curves use $\omega \tau=10$; the dotted curves are almost identical to the solid curves. The horizontal axis has been plotted using $z=d f / \nu_{l \infty}$. The horizontal dashed curve is the numerical evaluation of the real part of the analytical limit of $\nu \rightarrow \nu_{l}$

$$
\nu \rightarrow \nu_{l}=\nu_{l}^{\infty} \sqrt{\frac{\omega \tau}{2}}\left[F_{+}(\omega \tau)-j F_{-}(\omega \tau)\right]
$$

and the dashed curve with an initial value of $\nu=\nu_{s}$ is the numerical evaluation of the real part of the analytical limit of $d \rightarrow 0$ for the first Love wave mode. Considering the solid and dotted curves in Fig. 3, the effect on the real part of $\nu$ of including some viscoelasticity through the relaxation time while keeping both the frequency and $\nu_{l}^{\infty}$ constant appears to be relatively small. The transition in the dispersion curve in Fig. 3 between $\nu_{s}$ and $\nu_{l}^{\infty}$ occurring at $z \sim 1 / 4$ for the first mode and at $z \sim 3 / 4$ for the second mode, is sharpened, but the absolute changes in the real part of $\nu$ are small. To clarify this, the absolute changes in the real part of the phase velocity are shown in Fig. 4; in this figure the solid curve shows

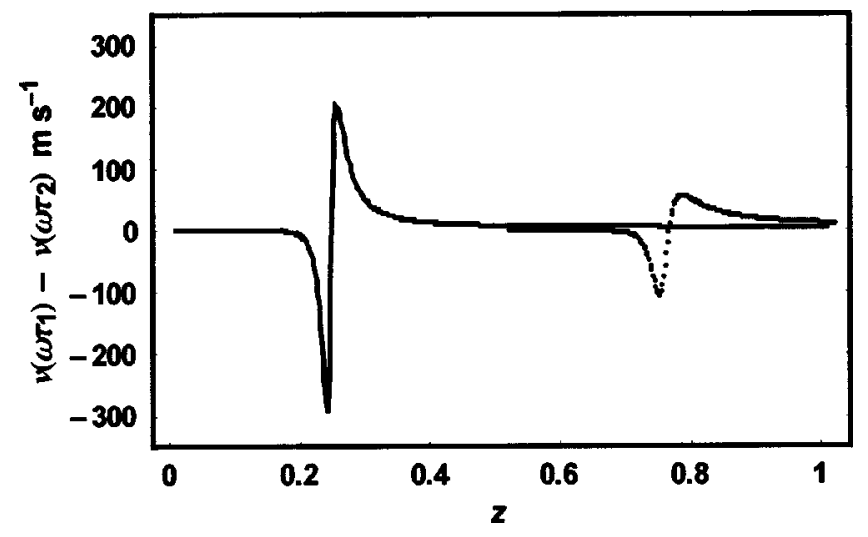

FIG. 4. The difference in the real part of the velocity as a function of the normalized guiding layer thickness $z=d f / \nu_{l}(\omega \tau \rightarrow \infty)$ for the data in Fig. 3; the solid curve is the difference for the first Love wave mode and the dotted curve is the difference for the second Love wave mode.

the first Love wave mode and the dotted curve shows the second Love wave mode. For the higher Love wave modes the value of $d$ at which the mode begins is also reduced as $\omega \tau$ decreases.

Figure 5, which plots the insertion loss as a function of the normalized guiding layer thickness $z$, shows that the effect of viscoelasticity on the insertion loss is considerably larger than on the real part of the velocity. In Fig. 5 a higher positive value indicates a signal that has greater loss and is, hence, weaker. The solid curve is the insertion loss per meter calculated using $\omega \tau=10^{6}$. The dotted curve is the insertion loss per meter scaled down by $10^{5}$ calculated using $\omega \tau$ $=10$; the scaling has been used to enable both curves to be displayed on the same diagram and has been chosen to be the ratio of the $\omega \tau$ 's. The accuracy of the numerical calculations for the insertion loss can be verified by considering the analytical limit for the insertion loss as $\nu \rightarrow \nu_{l}$ [i.e., Eq. (61)]. The insertion loss per meter is then given by

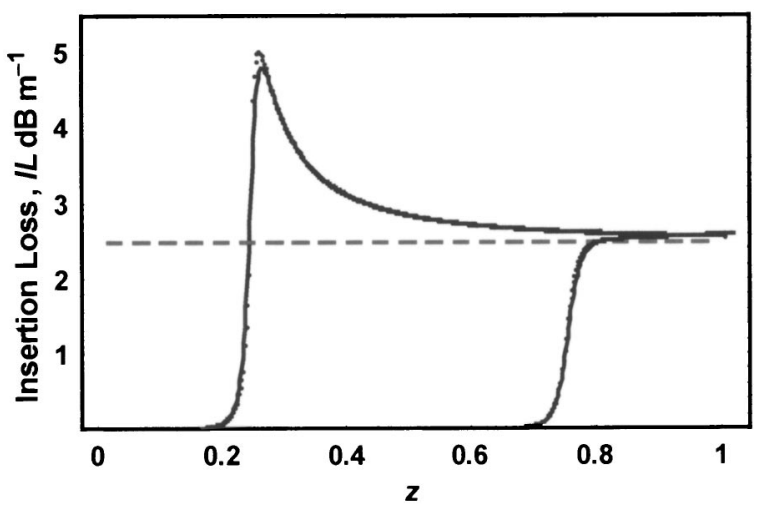

FIG. 5. The insertion loss per meter propagation path as a function of the normalized guiding layer thickness $z=d f / \nu_{l}(\omega \tau \rightarrow \infty)$ for the first two Love wave modes; the parameters are the same as in Fig. 3 . The solid curve is for $\omega \tau=10^{6}$ and the dotted curve is the insertion loss data for $\omega \tau=10$, but divided by a factor of $10^{5}$. The horizontal line is the $\nu \rightarrow \nu_{l}$ limit given by Eq. (62). 


$$
\begin{aligned}
\mathrm{IL} & =-20\left(\log _{10} e\right) \operatorname{Im}\left[\frac{\omega}{\nu}\right] \\
& \rightarrow 20\left(\log _{10} e\right)\left(\frac{\omega}{\nu_{l}^{\infty}}\right) \sqrt{\frac{1+(\omega \tau)^{2}}{2 \omega \tau}} F_{-}(\omega \tau)
\end{aligned}
$$

and this formula explains the choice of scaling used in presenting the numerical calculation for the curve with $\omega \tau$ $=10$. Provided $\omega \tau \geqslant 10$, the $F_{-}(\omega \tau)$ function can be approximated to $1 /(8 \omega \tau)^{3 / 2}$ thus giving $I L \propto 1 / \omega \tau$ in the limit $\nu \rightarrow \nu_{l}$.

Both the "low loss" $\omega \tau=10^{6}$ and "high loss" $\omega \tau=10$ curves in Fig. 5 show a characteristic change with increasing guiding layer thickness. Considering the first Love wave mode, for very thin guiding layers the insertion loss in both cases is small, but as the guiding layer thickness increases the loss increases significantly with a particularly rapid change occurring at around $z \sim 1 / 4$ for the first mode. Subsequently, the loss overshoots and then saturates at a constant value given by Eq. (62). A similar behavior occurs for the second Love wave mode, although the overshoot is hardly apparent and the particularly rapid change occurs at around $z \sim 3 / 4$. Thus, the insertion loss depends on the Love wave mode so that, for example, at a guiding layer thickness of $z$ $\sim 0.65$ the losses for the first Love wave mode are high, while the losses for the second Love wave mode are low. For the first Love wave mode, an overshoot can also be seen as $d \sim \lambda_{l}^{\infty} / 4$, where $\lambda_{l}^{\infty}=v_{l}^{\infty} / f$ and this can be shown to be due to the $\tan x$ term in Eq. (57). Indeed, it is similar in origin to the idea of a shear wave resonance known in work with QCMs. ${ }^{24,25}$ A close comparison of Eq. (36) in the thin layer mass loading limit with acoustic impedance models for QCMs shows that the $\tan x$ term in Eq. (57) is the term in the acoustic impedance that is the source of the shear wave resonance idea.

Physically, it is possible to understand the insertion loss behavior as a consequence of the transfer of the Love wave from a wave similar to a shear acoustic wave in the substrate to one similar to a shear acoustic wave in the guiding layer as the guiding layer thickness increases. In our previous treatment of Love waves with elastic mass guiding layers we plotted displacement profiles for a range of guiding layer thicknesses. ${ }^{20}$ In any mode the upper, free, surface of the guiding layer is an antinode and the displacement decays into the substrate. For the first Love wave mode $(n=0)$ and very thin guiding layers, the displacement in the substrate decays gently so that the substrate displacement approximates a plane wave and this plane wave pattern extends into the guiding layer. As the guiding layer thickness increases the displacement of the first Love wave mode $(n=0)$ becomes similar to a quarter wavelength type pattern in the guiding layer with the displacement decaying so rapidly into the substrate that the substrate-guiding layer interface almost becomes the location of a node in the displacement. Effectively, for thin guiding layers the Love wave is a shear acoustic wave in the substrate, with a wave velocity similar to a shear acoustic wave in the substrate $\nu_{s}$, and for thick guiding layers it is a wave in the guiding layer with a wave velocity similar to a shear acoustic speed in the layer $\nu_{l}$. For the next Love wave mode $(n=1)$, the wave begins as a plane wave in the substrate with a half-wavelength type pattern in the guiding layer and then evolves with increasing guiding layer thickness into an almost zero displacement in the substrate with a three-quarter wavelength displacement pattern in the guiding layer. Since the substrate is much thicker than the guiding layer the initial plane wave in the substrate represents the majority of the displacement and the half-wavelength pattern in the guiding layer is only a small part of the overall displacement. However, for the thicker guiding layers the substrate displacement almost vanishes and so the displacement in the layer is the dominating part of the overall wave displacement. In a manner similar to the first Love wave mode, the transition in the displacement pattern corresponds to a change of the wave velocity from $\nu_{s}$ to $\nu_{l}$. In this interpretation the insertion loss arising due to the viscoelasticity of the guiding layer would only become effective when the displacement is dominated by the displacement in the guiding layer and this only occurs once the transition in velocity toward the layer value $\nu_{l}$ occurs. Thus, we would expect the first Love mode to have significant damping at, for example, $d \sim 0.65$, while the second Love mode to simultaneously have little damping. Moreover, once the Love wave mode is localized into the guiding layer, the insertion loss would be expected to saturate, as is clearly the case from Fig. 5.

The prediction that one Love wave mode can have significant damping while the next higher Love wave can simultaneously have little damping is consistent with reported data. ${ }^{16}$ Frequency spectrums for Love wave devices show that as a guiding layer is built up systematically, the resonant frequency shifts to a lower value, and the wave eventually appears to be completely damped. However, data also show that if the guiding layer thickness is further increased, then after a given thickness a strong mode appears back at the original frequency and then again shifts with increasing guiding layer thickness to lower frequency until it is completely damped. This pattern has been observed through a sequence of more than seven Love wave modes. One practical caution against a too literal numerical application of our results to experimental data is that the theory concerns Love waves generated from SH-SAW supporting substrates. Data on Love waves taken using a SSBW mode would not have an insertion loss predicted using this theory because in the SSBW case, the guiding layer thickness also appears to decrease the angle at which the SSBW is launched into the substrate. For a Love wave generated from a SSBW mode, the initial effect of a guiding layer is to improve the transmission of the wave rather than to damp the wave. Eventually, as the wave localizes to the guiding layer the loss should become equal to that predicted by Eq. (62). Thus, the theory in this work may apply quantitatively to Love waves on a $36^{\circ} \mathrm{YZ}-\mathrm{LiTaO}_{3} \mathrm{SH}-\mathrm{SAW}$ substrate with the propagation path metallized, but only qualitatively to Love waves on a $90^{\circ}$ rotated ST-cut quartz SSBW substrate.

\section{Mass/liquid sensitivity}

In QCM sensors targeted at mass deposition applications, it is usual to quantify the mass sensitivity by the phase 


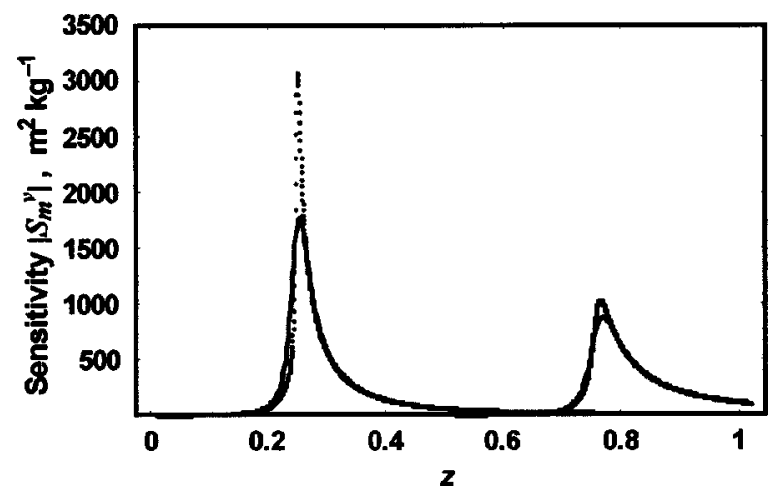

FIG. 6. Magnitude of the phase velocity mass sensitivity function, $\left|S_{m}^{\nu}\right|$, [i.e., Eq. (54) with $\nu_{f}=\nu_{l}$; ; the parameters are the same as in Fig. 2; The solid curve is for $\omega \tau=10^{6}$ and the dotted curve is the insertion loss data for $\omega \tau=10$.

velocity mass sensitivity [i.e., $S_{m}^{\nu}$ defined by Eq. (54) with $\rho_{f} \Delta h=\Delta m$ and $\left.\nu_{f}=\nu_{l}\right]$; to a first approximation the attenuation vanishes for thin mass layers. Figure 6 shows the magnitude of the phase velocity mass sensitivity for the data in Fig. 3 derived from the slope of the curves in Fig. 3; the solid curve corresponds to $\omega \tau=10^{6}$ and the dotted curve corresponds to $\omega \tau=10$. The effect of increasingly viscoelasticity (i.e., reducing $\omega \tau$ ) is to increase the peak sensitivity, although for thinner guiding layers the viscoelasticity can reduce the sensitivity. The reduction in the sensitivity prior to the peak may be important experimentally because the insertion loss increases with increasing viscoelasticity and it may not therefore be possible to operate a device at the guiding layer thickness required for peak phase velocity mass sensitivity.

In QCM sensors attenuation occurs if the mass deposited is viscoelastic, but not if the mass is purely elastic. Therefore, an important aspect of the insertion loss arising from viscoelasticity of the waveguiding in a Love wave device is that in theory it provides a highly sensitive sensor parameter both for liquid sensing and mass sensing even when the mass is purely elastic. To understand why, it is necessary to reconsider the origin of the high phase velocity mass sensitivity in a Love wave device with a guiding layer composed of elastic mass. Equation (44) shows that the phase velocity mass sensitivity is directly proportional to the slope of the logarithm of the mode velocity and that the highest phase velocity mass (and liquid) sensitivity occurs when a device is operated at the point of steepest slope on the dispersion curve. The point of steepest slope corresponds to the mode being on the point of transition between a wave dominantly in the substrate, and so having $v \sim v_{s}$, to one dominantly in the layer, and so having $v \sim v_{l}$. In a similar manner, in the case with viscoelasticity the insertion loss also changes from a value characteristic of the substrate (i.e., zero) to a value characteristic of the layer [i.e., given by Eq. (62)]. We would therefore expect high insertion loss mass and liquid sensitivity to correspond to the point of steepest slope of the insertion loss curve and for that slope to be particularly steep if the polymer induces high insertion losses. To be more quantitative, Eq. (19) for the insertion loss shows that the change in in- sertion loss per unit propagation length due to a small change in the (complex) phase velocity is

$$
\Delta \mathrm{IL}=20\left(\log _{10} e\right) \operatorname{Im}\left[\frac{\omega}{\nu_{0}}\left(\frac{\Delta \nu}{\nu_{0}}\right)\right]
$$

and the fractional shift in the phase velocity is given via Eq. (37) using the $g$ function or from Eq. (44) which uses the slope of $\log _{e} \nu$. In Eq. (63) a positive $\Delta \mathrm{IL}$ represents a weaker signal.

In the approximation that the material being sensed is elastic mass with a shear acoustic velocity similar to that of the guiding-layer (i.e., $\nu_{f} \approx \nu_{l}$ ), then Eqs. (44) and (63) predicts that the maximum change in insertion loss for a given deposited mass ( $\Delta m=\rho_{f} h$ with $h$ small) will be high when the operating point is chosen such that the imaginary part of $\nu_{0}^{-1} d \log _{e} \nu / d z$ is large. For large relaxation times, this combination is dominated by the imaginary part of the slope $d \log _{e} \nu / d z$. Thus, a key conclusion is that when using a Love wave device to sense rigidly coupled elastic mass, insertion loss can be a highly sensitive parameter because the elastic mass can convert the wave from having a low loss to a high loss via the transfer of the displacement from the substrate to the guiding layer; there is no absolute requirement for the deposited mass itself to be viscoelastic. While, in a sense, the elastic mass does not itself have a loss, the mass effectively moves the operating point of the device down the complex dispersion curve into a region where the guiding layer losses start to dominate the Love wave.

For sensing rigidly coupled elastic mass of shear acoustic velocity $v_{m}$, deposited from vacuum, we can define an insertion loss mass sensitivity function $S_{m}^{\mathrm{IL}}$ in a manner similar to the phase velocity mass sensitivity function using the change in insertion loss per meter divided by the mass per unit area

$$
\begin{aligned}
S_{m}^{\mathrm{IL}}= & \lim _{\Delta m \rightarrow 0}\left(\frac{\Delta \mathrm{IL}}{\Delta m}\right) \\
= & 20\left(\log _{10} e\right) \operatorname{Im}\left[\frac{\omega^{2}}{2 \pi \rho_{l} \nu_{l}^{\infty}}\left(\frac{1-\nu_{m}^{2} / \nu_{0}^{2}}{1-\nu_{l}^{2} / \nu_{0}^{2}}\right)\right. \\
& \left.\times \frac{1}{\nu_{0}}\left(\frac{d \log _{e} \nu}{d z}\right)_{z=z_{0}}\right],
\end{aligned}
$$

where Eqs. (44) and (63) have been used to obtain the relationship to the slope of the dispersion curve. One immediate consequence of Eq. (64) is that the peak sensitivity in the insertion loss does not necessarily occur at the same operating point on the dispersion curve as the peak sensitivity in phase velocity. For example, when sensing a mass layer composed of approximately the same material as the guiding layer, the peak phase velocity sensitivity will correspond to the maximum of $d \log _{e} \nu / d z$, while the peak insertion loss sensitivity will correspond to the maximum of $\nu^{-1} d \log _{e} \nu / \mathrm{dz}$. Figure 7 shows the insertion loss mass sensitivity for a Love wave with a viscoelastic guiding layer with the same parameters as used for the data in Fig. 3; the approximation $\left(1-\nu_{m}^{2} / \nu_{0}^{2}\right) /\left(1-\nu_{l}^{2} / \nu_{0}^{2}\right) \ll 1$ has been used in 


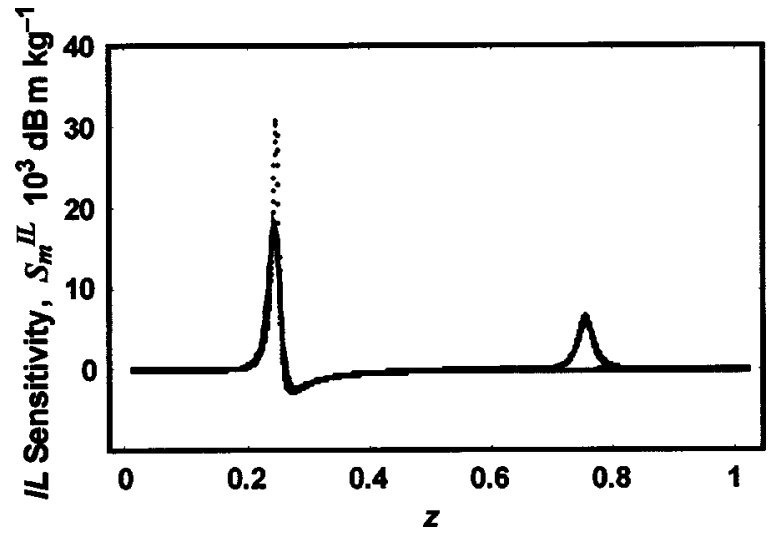

FIG. 7. The insertion loss mass sensitivity, $S_{m}^{\mathrm{IL}}$, for sensing material of the same type as the guiding layer [i.e., Eq. (64) with $\nu_{m}=\nu_{l}$ ]; the parameters are the same as in Fig. 2. The solid curve is for $\omega \tau=10^{6}$ and the dotted curve is the insertion loss data for $\omega \tau=10$, but divided by a factor of $10^{5}$.

Eq. (64). The solid curve corresponds to $\omega \tau=10^{6}$ and the dotted curve corresponds to $\omega \tau=10$, but it should also be noted that the data for $\omega \tau=10$ has been scaled down by a factor of $10^{5}$ in order to plot the figures on the same diagram for comparison of their shapes. The need to use a large scaling factor in presenting the data for the effect of viscoelasticity on the insertion loss mass sensitivity emphasizes that experimentally insertion loss may be a very useful Love wave sensor parameter; we would also expect this to be true for other layer-guided acoustic wave sensors.

If the Love wave device is being used to sense changes due to the device being immersed in a liquid, then Eqs. (44) and (45) show that insertion losses will arise whether or not the guiding layer is viscoelastic. In the infinitely deep Newtonian liquid case given by Eq. (45), $\tan \left(T_{f}^{0} h\right) / T_{f}^{0} h$ has real and imaginary components of equal magnitude [since $\left.(-2 j)^{1 / 2}=1-j\right]$ and a Love wave device with an elastic guiding layer will couple the imaginary part into an insertion loss. If the guiding layer becomes a viscoelastic material, then the real part of $\tan \left(T_{f}^{0} h\right) / T_{f}^{0} h$ will also become coupled into the insertion loss via the imaginary part of the slope of $\log _{e} v_{0}$ (multiplied by $1 / v_{0}$ ). This additional mechanism for insertion loss changes, introduced by the viscoelasticity of the guiding layer, may provide even higher sensitivity in liquid phase sensing applications. The mass sensitivity defined by Eq. (64) does not include this additional mechanism for the liquid phase sensitivity. However, because for the infinitely deep Newtonian liquid the real and imaginary contributions of $\tan \left(T_{f}^{0} h\right) / T_{f}^{0} h$ have equal magnitude, the relative importance of the viscoelasticity of the guiding layer to the insertion loss can be assessed by considering the relative magnitudes of the real and imaginary parts of the slopes of $\log _{e} v$ and this is shown in Fig. 8. The parameters used in Fig. 8 are the same as for the data in Fig. 2; the solid curves corresponds to $\omega \tau=10^{6}$ and the dotted curves correspond to $\omega \tau=10$. The curves with negative peak values are the real part of the slope and the curves with positive peak values are the imaginary part of the slope. It is evident from the existence of only dotted curves with positive peak values for the two Love wave modes in Fig. 8 that the imaginary part of the

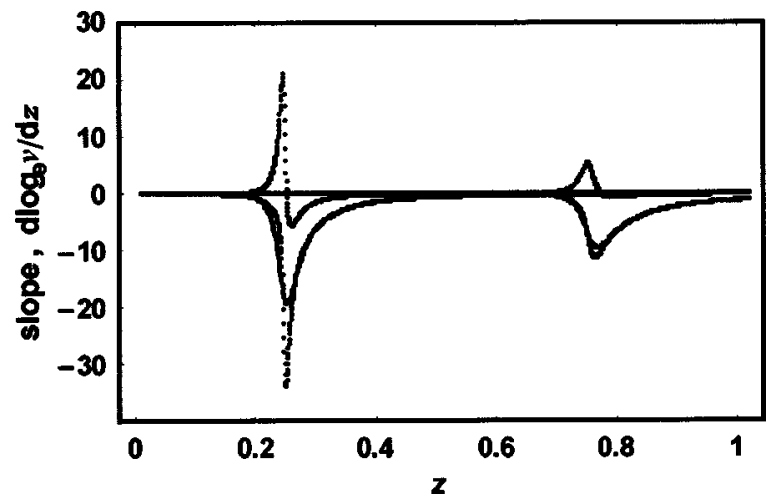

FIG. 8. Comparison of the real and imaginary parts of $d \log _{e} \nu / d z$ for the data in Fig. 3. The dotted curves with positive peaks are the imaginary part of $d \log _{e} \nu / d z$ for $\omega \tau=10$; the corresponding data for $\omega \tau=10^{6}$ is vanishingly small on the scale of the figure. The solid and dotted curves with negative peaks are the real part of $d \log _{e} \nu / d z$ for $\omega \tau=10^{6}$ and $\omega \tau=10$, respectively. Data for the first two Love modes are shown.

slope is vanishingly small for $\omega \tau=10^{6}$. However, the real part of the slope (curves with negative peak values) is of comparable order of magnitude for both $\omega \tau=10^{6}$ and $\omega \tau$ $=10$. Thus, for liquid phase sensing we would expect two contributions to arise from the slope (i.e., $d \log _{e} \nu / d z$ ) to the phase velocity sensitivity as the guiding layer becomes viscoelastic. For liquid phase sensing similar conclusions also apply for the insertion loss sensitivity although the relevant factor is $\nu^{-1} d \log _{e} \nu / d z$ rather than simply the slope $d \log _{e} \nu / d z$.

In this section we have not considered numerically the effect of depositing mass from the liquid phase because this requires a substantially more difficult root finding procedure based on Eq. (47) [or Eq. (50)] rather than the simpler Eq. (36). However, some qualitative comments are possible. The simplest view of the liquid phase is that it has two effects: first the liquid shifts downwards each point of the dispersion curve for the device in vacuum to create a new dispersion curve, and second, the magnitude of the slope of the curve at the operating point is subsequently higher. The idea that the vacuum based device response to liquid can simply be added to the vacuum based device response to the mass uses the assumption that the change in slope of the dispersion curve can be neglected. Since a Love wave device has high phase velocity mass sensitivity precisely because it is operated at a point of steep slope on the dispersion curve, it is far from obvious that the assumption that the change in slope can be neglected will be true. Moreover, for a viscoelastic guiding layer it is difficult to predict qualitatively how the introduction of the imaginary part to the slope will change the response. Nonetheless, the equations developed in this work provide a rigorous basis for a numerical investigation of these issues. We would anticipate that the insertion loss response will be particularly important in biological sensing applications were the mass being sensed often has an element of viscoelasticity and is usually deposited from the liquid phase. 


\section{CONCLUSION}

A theoretical treatment of Love waves on finite substrates and with viscoelastic wave-guiding layers, and of shear horizontal acoustic plate modes with coatings, has been developed. Equations for the sensor response of these types of systems for both mass and viscoelastic or Newtonian liquid phase applications have been derived and the limiting cases of mass and liquid response for shear horizontal polarized surface acoustic wave and shear horizontal acoustic plate mode sensors detailed. The response of Love wave and layer-guided SH-APMs to a general material (elastic mass, Newtonian liquid, or viscoelastic fluid) has been shown to depend on the slope of the complex dispersion curve and the relationship to the QCM mass and liquid phase sensor response has been discussed. Equations describing mass deposition from the liquid phase have been developed. The analytical results have been investigated using numerical calculations, based on a Maxwell model of viscoelasticity for the waveguiding layer of Love wave devices. The role of the waveguiding layer's viscoelasticity in creating an insertion loss and modifying the mass sensitivity has been quantified. It has been suggested that, by using a viscoelastic material as a waveguiding layer, insertion loss can be a useful sensor parameter for studying not only liquid phase response, but also mass deposition response.

\section{ACKNOWLEDGMENTS}

Two of the authors (G.M. and M.I.N.) gratefully acknowledge the BBSRC for financial support under Research Grant No. 301/E11140.

\section{APPENDIX A: COMPARISON TO LITERATURE SH-APM FORMULAE}

To compare our result for the perturbation of a SH-APM sensor response by a viscoelastic liquid to the result from Martin et al. ${ }^{22}$ we start from our Eq. (33)

$$
\left(\frac{\Delta \nu}{\nu_{m}}\right) \approx \frac{\sqrt{-2 j} \eta_{f} \nu_{m}^{2}}{\nu_{s}^{2} \rho_{s} w \omega(1+j \omega \tau) \alpha_{m}} .
$$

From Martin et al.'s definition of $\gamma_{m}$ we can find that $\gamma_{m}^{2}$ $=2 j / \alpha_{m}^{2}$ and so Eq. (A1) becomes

$$
\left(\frac{\Delta \nu}{\nu_{m}}\right) \approx \frac{2 \nu_{m}^{2}}{\nu_{s}^{2} \rho_{s} w}\left[\frac{\eta_{f}}{2 \omega}\right]\left[\frac{j \gamma_{m}}{(1+j \omega \tau)}\right]
$$

and it has a real part of

$$
\begin{aligned}
\left(\frac{\Delta \nu}{\nu_{m}}\right) & \approx \frac{2 \nu_{m}^{2}}{\nu_{s}^{2} \rho_{s} w}\left[\frac{\eta_{f}}{2 \omega}\right] \operatorname{Re}\left[\frac{j \gamma_{m}}{(1+j \omega \tau)}\right] \\
& =\frac{-2 \nu_{m}^{2}}{\nu_{s}^{2} \rho_{s} w}\left[\frac{\eta_{f}}{2 \omega}\right] \operatorname{Im}\left[\frac{\gamma_{m}}{(1+j \omega \tau)}\right] .
\end{aligned}
$$

Defining a constant $c$ by

$$
c=\frac{2 v_{m}^{2}}{v_{s}^{2} \rho_{s} w}
$$

and comparing to Eqs. (B11) and Eq. (B12) gives the same formula for the perturbation in velocity as in Martin et al. provided our constant $c$ is replaced by their constant $c_{\nu}$ defined as

$$
c_{\nu}=\left(\frac{\nu_{m}}{\nu_{g m}}\right) \frac{J_{m}}{\rho_{s} w},
$$

where $J_{m}=1$ since $m>0$; in our work we have previously indicated that the $m=0$ result quoted by Martin et al. assumes that the SH-APM is not converted to a Love wave by the perturbing material. Thus, for $m>0$ the only difference between our SH-APM perturbation formula and that of Martin $e t a l$. is the replacement of a mode group velocity $\nu_{g m}$ by our $\nu_{s}^{2} / 2 \nu_{m}$. The insertion loss for the damping of a $\mathrm{SH}-$ APM sensor by a viscoelastic liquid of infinite depth arising from our velocity perturbation formula is

$$
\mathrm{IL} \approx-\left(\frac{20 \log _{10} e}{2 \pi \sqrt{2}}\right)\left(\frac{\omega L}{\rho_{s} \nu_{s}^{2}}\right)\left(\frac{2 \pi \nu_{m}}{W \omega}\right) \sqrt{\omega \eta_{f} \rho_{f}} F_{+}(\omega \tau),
$$

whereas the formula from the Martin et al. article would have an additional factor of $c_{\nu} / c$. A similar formula for the damping was also given by Ricco and Martin in an earlier article, ${ }^{23}$ but in that case the third factor in brackets in Eq. (A6) was absent. Our first factor in brackets in Eq. (A6) evaluates to 0.9775 , the second term is their $A$ factor, and the $F_{+}(\omega \tau)$ is their $F(\omega \tau)$ function. The primary difference between our insertion loss formula for the viscoelastic liquid perturbation of an SH-APM sensor and the Ricco and Martin formula ${ }^{23}$ is the existence of the third factor, which evaluates to around 0.17 for the lowest modes for the data in their article. It is not obvious from the published work why this factor should have been taken as unity. One argument might be to argue that $\left(2 \pi \nu_{m} / W \omega\right)=\left(\nu_{m} / W f\right)$ and assume $\nu_{m}$ $=f \lambda_{m}$ so that the factor becomes $\lambda_{m} / W$ and then approximate it to unity. For example, by taking $W \sim m \lambda_{m} / 2$ and averaging $m=1,2$, and $3 \lambda_{m} / W$ gives 11/9. However, such an argument would be wrong because it implies $w \sim m \nu_{m} / 2 f$ rather than the relationship among the substrate thickness, mode speed, and frequency given by Eq. (32).

\section{APPENDIX B: FOUR-LAYER MODEL}

The layers in the model are defined in Fig. 2 and are the substrate, guiding layer, mass layer and fluid layer, or thicknesses, $w, l, m$, and $f$, respectively; subscripts $s, l, m$, and $f$ are used to indicate quantities related to these layers. The displacements of the layers are given by

$$
\begin{aligned}
& \underline{u_{s}}=(0,1,0)\left\lfloor C_{s} e^{-T_{s} x_{3}}+D_{s} e^{T_{s} x_{3}}\right\rfloor e^{j\left(\omega t-k_{1} x_{1}\right)}, \\
& \underline{u_{k}}=(0,1,0)\left\lfloor A_{k} e^{-j T_{k} x_{3}}+B_{k} e^{j T_{k} x_{3}}\right\rfloor e^{j\left(\omega t-k_{1} x_{1}\right)},
\end{aligned}
$$

and the wave vectors by

$$
\begin{aligned}
& T_{s}^{2}=\omega^{2}\left(\frac{1}{\nu^{2}}-\frac{1}{\nu_{s}^{2}}\right), \\
& T_{k}^{2}=\omega^{2}\left(\frac{1}{\nu_{k}^{2}}-\frac{1}{\nu^{2}}\right),
\end{aligned}
$$


where $k=l, m$, or $f$ in Eqs. (B2) and (B4) so that each represents three equations. To obtain the dispersion equation it is necessary to impose boundary conditions of continuity of stress and displacement at each interface between layers and of the vanishing of stress at the two free surfaces. The continuity of the displacements at the boundaries between the substrate and guiding layer, guiding layer and mass layer, and mass layer and fluid layer give

$$
\begin{aligned}
& C_{s}+D_{s}=A_{l}+B_{l}, \\
& \begin{aligned}
A_{l} \exp \left(-j T_{l} d\right)+B_{l} \exp \left(j T_{l} d\right) \\
\quad=A_{m} \exp \left(-j T_{m} d\right)+B_{m} \exp \left(j T_{m} d\right), \\
A_{m} \exp \left(-j T_{m}(d+h)\right)+B_{m} \exp \left(j T_{m}(d+h)\right) \\
\quad=A_{f} \exp \left(-j T_{f}(d+h)\right)+B_{f} \exp \left(j T_{m}(d+h)\right) .
\end{aligned}
\end{aligned}
$$

In addition, the stress boundary conditions at the two free surfaces give

$$
C_{s} \exp \left(T_{s} w\right)-D_{s} \exp \left(-T_{s} w\right)=0,
$$

$$
\begin{aligned}
A_{f} \exp \left(-j T_{f}(d+h+b)\right) & \\
& -A_{f} \exp \left(j T_{f}(d+h+b)\right) \\
= & 0
\end{aligned}
$$

and the continuity of stress provides a further three equations

$$
C_{s}-D_{s}=j \xi_{l m}\left(A_{l}-B_{l}\right) \text {, }
$$

$A_{l} \exp \left(-j T_{l} d\right)-B_{l} \exp \left(j T_{l} d\right)$

$$
=\xi_{m l}\left[A_{m} \exp \left(-j T_{m} d\right)-B_{m} \exp \left(j T_{m} d\right)\right],
$$

$$
\begin{aligned}
& A_{m} \exp \left(-j T_{m}(d+h)\right)-B_{m} \exp \left(j T_{m}(d+h)\right) \\
& \quad=\xi_{f m}\left\lfloor A_{f} \exp \left(-j T_{f}(d+h)\right)-B_{f} \exp \left(j T_{m}(d+h)\right)\right\rfloor,
\end{aligned}
$$

where $\xi_{i j}=G_{i} T_{i} / G_{j} T_{j}$ and the $G_{i}$ s are the complex shear modulii which are defined by Eq. (14) for the Maxwell model of viscoelasticity. Solving the eight equations Eqs. (B5)-(B12) gives the full dispersion equation for the fourlayer system

$$
\xi_{f m} \tan \left(T_{f} b\right)=\frac{\left[\xi_{l m} \tan \left(T_{l} d\right)-\xi_{s m} \tanh \left(T_{s} w\right)\right]+\tan \left(T_{m} h\right)\left[1+\xi_{s l} \tanh \left(T_{s} w\right) \tan \left(T_{l} d\right)\right]}{\tan \left(T_{m} h\right)\left[\xi_{l m} \tan \left(T_{l} d\right)-\xi_{s m} \tanh \left(T_{s} w\right)\right]-\left[1+\xi_{s l} \tanh \left(T_{s} w\right) \tan \left(T_{l} d\right)\right]},
$$

which has the correct limits for the systems of a simple substrate, substrate plus layer, and substrate plus two layers. In order to develop the model for the effect of mass deposition from a liquid Eq. (B13) is rewritten with the terms involving the mass layer grouped together

$$
\begin{aligned}
{\left[\tan \left(T_{l} d\right)-\xi_{s l} \tanh \left(T_{s} w\right)\right]+\xi_{f l} \tan \left(T_{f} b\right) } \\
\quad \times\left[1+\xi_{s l} \tanh \left(T_{s} w\right) \tan \left(T_{l} d\right)\right] \\
=\tan \left(T_{m} h\right)\left\{\left[\tan \left(T_{l} d\right)-\xi_{s l} \tanh \left(T_{s} w\right)\right] \xi_{f m}\right. \\
\left.\quad \times \tan \left(T_{f} b\right)-\left[1+\xi_{s l} \tanh \left(T_{s} w\right) \tan \left(T_{l} d\right)\right] \xi_{m l}\right\} .
\end{aligned}
$$

When $h=0$, the left-hand side of Eq. (B14) equals zero and defines the unperturbed system of a device composed of a substrate and waveguiding layer immersed in a viscoelastic fluid of thickness $b$. In Eq. (B14), the dependence on the perturbing mass occurs through the $\tan \left(T_{m} h\right), \xi_{f m}$, and $\xi_{f m}$ factors on the right-hand side of the equation. Noting that $\mu_{m} T_{m}{ }^{02}=\rho_{m} \omega^{2}\left(1-\nu_{m}^{2} / \nu_{0}^{2}\right)$ and performing an expansion about $h=0$, we find

$$
\begin{aligned}
\frac{\Delta \nu}{\nu_{0}} \approx & g_{L}\left(\omega, \nu_{0}, \text { substrate, guiding layer, fluid }\right) \\
& \times\left(1-\frac{\nu_{m}^{2}}{\nu_{0}^{2}}\right)\left(1+\xi_{f m}^{2} \tan ^{2}\left(T_{f}^{0} b\right)\right) \rho_{m} \Delta h,
\end{aligned}
$$

where $g_{L}$ is a function depending on the operating point of the unperturbed system.

${ }^{1}$ D. S. Ballantine, R. M. White, S. J. Martin, A. J. Ricco, E. T. Zellers, G. C. Frye, and H. Wohjlten, Acoustic Wave Sensors (Academic, New York, 1997).
${ }^{2}$ M. Thompson and D. C. Stone, Surface-Launched Acoustic Wave Sensors (Wiley, New York, 1997).

${ }^{3}$ M. J. Vellekoop, Ultrasonics 36, 7 (1998).

${ }^{4}$ B. A. Martin and H. E. Hager, Anal. Chem. 67, 3324 (1995).

${ }^{5}$ R. M. White, Faraday Discuss. 107, 1 (1997).

${ }^{6}$ G. Z. Sauerbrey, Z. Phys. 155, 206 (1959).

${ }^{7}$ H. L. Bandey, S. J. Martin, and R. W. Cernosek, Anal. Chem. 71, 2205 (1999)

${ }^{8}$ K. K. Kanazawa and J. G. Gordon, II, Anal. Chim. Acta 175, 99 (1985).

${ }^{9}$ D. P. Morgan, Surface-Wave Devices for Signal Processing (Elsevier, New York, 1991)

${ }^{10}$ C. K. Campbell, Surface Acoustic Wave Devices for Mobile and Wireless Communications (Academic, New York, 1998).

${ }^{11}$ E. Benes, M. Gröschl, F. Seifert, and A. Pohl, IEEE Trans. Ultrason. Ferroelectr. Freq. Control 45, 1314 (1998).

${ }^{12}$ E. Gizeli, A. C. Stevenson, N. J. Goddard, and C. R. Lowe, IEEE Trans. Ultrason. Ferroelectr. Freq. Control 39, 657 (1992).

${ }^{13}$ G. Kovacs and A. Venema, Appl. Phys. Lett. 61, 639 (1992).

${ }^{14}$ J. Du and G. L. Harding, Smart Mater. Struct. 6, 716 (1997).

${ }^{15}$ E. Gizeli, Smart Mater. Struct. 6, 700 (1997)

${ }^{16}$ G. McHale, M. I. Newton, F. Martin, K. A. Melzak, and E. Gizeli, Appl. Phys. Lett. 79, 3542 (2001).

${ }^{17}$ C. J. Percival, S. Stanley, A. Braithwaite, M. I. Newton, and G. McHale, Analyst 127, 1024 (2002).

${ }^{18}$ C. J. Percival, S. Stanley, M. Galle, A. Braithwaite, M. I. Newton, G. McHale, and W. Hayes, Anal. Chem. 73, 6995 (2001).

${ }^{19}$ M. I. Newton, G. McHale, F. Martin, E. Gizeli, and K. A. Melzak, Europhys. Lett. 58, 818 (2002).

${ }^{20}$ G. McHale, M. I. Newton, and F. Martin, J. Appl. Phys. 91, 5735 (2002).

${ }^{21}$ G. McHale, M. I. Newton, and F. Martin, J. Appl. Phys. 91, 9701 (2002).

${ }^{22}$ S. J. Martin, A. J. Ricco, T. M. Niemczyk, and G. C. Frye, Sens. Actuators 20, 253 (1989).

${ }^{23}$ A. J. Ricco and S. J. Martin, Appl. Phys. Lett. 50, 1474 (1987).

${ }^{24}$ G. McHale, M. K. Banerjee, M. I. Newton, and V. V. Krylov, Phys. Rev. B B59, 8262 (1999).

${ }^{25}$ S. J. Martin, H. L. Bandey, R. W. Cernosek, A. R. Hillman, and M. J. Brown, Anal. Chem. 72, 141 (2000). 